\title{
Gestörte Kaufunktion
}

\section{Wiederherstellende Verfahren bei gestörter Kaufunktion}

\section{W. B. Freesmeyer \\ M. R. Fussnegger ${ }^{1}$ \\ M. O. Ahlers ${ }^{2}$}

\section{Inhaltsverzeichnis}

1 Einleitung ...249

2 Hals-, Nasen-, Ohrenärztliche Faktoren bei CMD ...249

2.1 Otalgien...249

2.2 Tinnitus ... 250

2.3 Sinusitiden ...251

2.4 Eagle-Syndrom ...251

2.5 Überweisung zum Zahnarzt...251

3 Diagnostik ...251

3.1 Basisdiagnostik: Funktioneller Kurzbefund als CMD-Screening ... 251

3.2 Erweiterte Diagnostik: Klinische Funktionsanalyse ... 252

3.2.1 Anamnese...252

3.2.2 Schmerzlokalisation ... 252

3.2.3 Palpation...252

3.2.4 Okklusionsdiagnostik...253

3.2.5 Neurologische Kurzuntersuchung ...253

3.2.6 Orthopädische Kurzuntersuchung...253

3.3 Weiterführende Diagnostik...253

3.3.1 Manualmedizinische Strukturanalyse ...253

3.3.2 Instrumentelle Diagnostik... 254

3.3.3 Bildgebende Diagnostik... 254

3.4 Auswertung: Diagnoseschema ...256
4 Therapie...256

4.1 Aufklärung...257

4.2 Selbstbeobachtung... 257

4.3 Entspannungstechniken ...257

4.4 Stressmanagement, Verhaltenstherapie ... 257

4.5 Physiotherapie...258

4.6 Medikamentöse Therapie ... 258

4.7 Schienentherapie...258

4.8 Okklusale Therapie ... 259

$5 \quad$ Schlussfolgerung ...260

Literatur (Hinweis: erscheint nur in der Online-Ausgabe)

Institutsangaben

${ }^{1}$ Charité - Universitätsmedizin Berlin, Campus Universitätsklinikum Benjamin Franklin, Klinik und Poliklinik für Zahn-, Mund- und Kieferheilkunde, Abt. für Restaurative Zahnmedizin

${ }^{2}$ Universitätsklinikum Hamburg-Eppendorf, Zentrum für Zahn-, Mund- und Kieferheilkunde, Poliklinik für Zahnerhaltung und Präventive Zahnheilkunde

Korrespondenzadresse

Professor Dr. Wolfgang B. Freesmeyer · Charité - Universitätsmedizin Berlin · Campus Universitätsklinikum Benjamin Franklin · Klinik und Poliklinik für Zahn-, Mund- und Kieferheilkunde · Abt. für Restaurative Zahnmedizin·Aßmannshauser Straße 4-6·14197 Berlin·E-mail: wolfgang.freesmeyer@charite.de 


\section{Zusammenfassung}

„Craniomandibuläre Dysfunktionen“ (CMD) bezeichnen Erkrankungen der Zähne, Parodontien, der Kaumuskulatur, der Kiefergelenke und mit diesen verbundene Strukturen. In den letzten Jahren hat sich gezeigt, dass neben anatomischen, physiologischen, parafunktionellen und anderen biologischen Ursachen auch psychische, soziale und allgemeinmedizinische Einflüsse eine enorme Bedeutung in der Ätiologie der CMD besitzen. Dies bedeutet, dass der mit einer CMD-Erkrankung konfrontierte Therapeut frühzeitig andere Fachdisziplinen, insbesondere Schmerztherapeuten, Neurologen, HNO-Ärzte aber auch Physiotherapeuten, sowie Psychotherapeuten hinzuziehen sollte. Die Notwendigkeit einer konsiliarischen Überweisung zum CMDfachkundigen und funktionsanalytisch tätigen Zahnarzt ist immer dann gegeben, wenn sich in der HNO-ärztlichen Untersuchung keine pathologischen Befunde ergeben. Die Therapie von CMD gliedert sich in folgende Schritte, die sich immer auf die zugrundeliegenden Diagnosen beziehen: Aufklärung des Patienten, Selbstbeobachtung, Entspannungstherapie, Verhaltenstherapie, Physiotherapie, medikamentöse Therapie, therapeutische Lokalanästhesien, Schienentherapie und gegebenenfalls einer prothetischen oder kieferorthopädischen Therapie um eine stabile Okklusion wiederherzustellen.

\section{Schlüsselwörter}

Craniomandibuläre Dysfunktion • biopsychosoziale Erkrankung · Funktionsdiagnostik · Funktionstherapie · Tinnitus

\section{$1 \quad$ Einleitung}

Störungen der Kaufunktion werden heute unter dem Begriff „Craniomandibuläre Dysfunktionen“ (CMD) zusammengefasst. Die Erkrankungen betreffen die Zähne, die Kaumuskulatur, die Kiefergelenke, Parodontien und mit diesen verbundene Strukturen, Nerven, Ohren, Hals, Kopf und die Wirbelsäule. Als Ursachen kommen unter anderem Stress und parafunktionelle Ursachen wie Knirschen und Pressen in Betracht. Auch Habits wie Lippenkauen oder Zungenpressen und Störungen im neurophysiologischen Zusammenspiel zwischen der Funktion der Zahnreihen, der Muskulatur und den Kiefergelenken können zu CMD beitragen. In den letzten Jahren hat sich die Betrachtung der Pathogenese funktioneller Symptome und Erkrankungen sowohl im Bereich der Diagnostik, der Therapie als auch bezüglich des Gesamtverständnisses bezüglich der Entstehung dieser Symptomund Krankheitsbilder geändert. In den Anfängen der Funktionsdiagnostik bestand hinsichtlich der Entstehung von CMD eine eher monokausale Betrachtungs- und Handlungsweise, die von einem einfachen Ursache-Wirkungs-Prinzip ausging:

Okklusionsstörung = Parafunktion

Parafunktion $=$ funktionelle Symptome und Erkrankungen durch hohe Belastungen an den Determinanten des craniomandibulären Systems.
In den letzten Jahren hat sich jedoch gezeigt, dass man mit dieser Betrachtungsweise vielen Patienten nicht gerecht wurde und der Behandlungserfolg bei manchen auch ganz ausblieb. Wie auch in anderen medizinischen Gebieten, z. B. beim chronischen Rückenschmerz, wurde erkannt, dass viele verschiedene Faktoren bei der Entstehung solcher Symptome und Erkrankungen eine Rolle spielen. Begriffe wie „Bio-psycho-soziale Erkrankung“ oder „Multifaktorielle Erkrankung“ geben diese Sichtweise wieder. Sie sollen zeigen, dass neben anatomischen, physiologischen, parafunktionellen und anderen biologischen Ursachen auch psychische und soziale Einflüsse eine enorme Bedeutung in der Ätiologie der CMD besitzen. Dies bedeutet, dass der mit einem CMDPatienten konfrontierte Zahnarzt oder Arzt bei Patienten, die unter nicht eindeutig zu verifizierenden oder chronischen Symptomen leiden, nicht mehr als Alleinbehandler arbeiten kann, sondern frühzeitig, in Abhängigkeit von der Initialdiagnose andere Fachdisziplinen, insbesondere Schmerztherapeuten, Neurologen, HNO-Ärzte, Orthopäden und Physiotherapeuten aber auch Psychologen konsultieren muss [1]. Darüber hinaus müssen Erkenntnisse der Neuro- und Schmerzphysiologie, z.B. der Schmerzchronifizierung in die Diagnostik und Therapie miteinbezogen werden. Man hat zudem aus der Schmerzmedizin gelernt, dass speziell chronische Schmerzen bei den betroffenen Patienten kein Warnsymptom einer zugrundeliegenden einfachen kausalen Ursachenkette mehr darstellen, sondern Schmerzzustände im craniomandibulären System sich auch als eigenständige Erkrankungen etablieren können [2]. Darüber hinaus ist es wünschenswert, dass aus wissenschaftlichen, ethischen, wie auch aus Kostengründen, reliable und validierte diagnostische und therapeutische Verfahren zum Einsatz kommen, um die Diagnostik und Therapie auf eine noch sicherere Grundlage zu stellen.

\section{Hals-, Nasen-, Ohrenärztliche Faktoren bei CMD}

Seit den 30er-Jahren des vergangenen Jahrhunderts werden immer wieder Beziehungen zwischen Kieferbeschwerden und Ohrsymptomen beschrieben. Das bekannteste Beschwerdebild wurde dabei das zum Teil heute noch im Sprachgebrauch befindliche „Costen-Syndrom“. Der gleichnamige Arzt beschrieb einen Zusammenhang, wonach sich nach einem Verlust von Stützzonen, also beispielsweise mehreren Molaren, eine Dorsal- und Kranialverlagerung des Unterkiefers einstellen könnte. Gelenknahe Strukturen wie der N. auriculotemporalis und die Tuba Eustachii könnten nach diesen Überlegungen irritiert werden und so zu Symptomen wie Otalgien, Tinnitus, Vertigo oder Geschmacksstörungen führen. Diese Theorie wurde jedoch mehrfach widerlegt und gilt heute als eine Vermischung verschiedener Krankheitsbilder. Der Terminus „Costen-Syndrom“ sollte aus diesem Grunde nicht mehr verwendet werden.

\section{$2.1 \quad$ Otalgien}

Tatsächlich beschreiben jedoch viele Patienten mit CMD Ohrsymptome, überwiegend dabei Ohrschmerzen, so dass vor der zahnärztlichen, funktionellen Untersuchung bereits häufig eine HNO-ärztliche Untersuchung, oftmals ohne pathologische Befunde durchgeführt wurde. Sehr oft ist die Ursache also nicht primär im Bereich des Ohres sondern vielmehr in unterschiedlichen orofazialen Strukturen zu suchen. Eine Auswahl ist in Tab. 1 auf- 
Tab. 1 Auswahl an akuten und chronischen, nicht otogenen Erkrankungen im orofazialen Bereich, die Ohrenschmerzen bewirken können

\begin{tabular}{|ll}
\hline akute, nicht otogene Erkrankungen & $\begin{array}{l}\text { chronische, nicht otogene } \\
\text { Erkrankungen }\end{array}$ \\
\hline chronische Pulpitis & $\begin{array}{l}\text { myofasziale Schmerzen der } \\
\text { Kaumuskulatur } \\
\text { myofasziale Schmerzen der } \\
\text { Nackenmuskulatur }\end{array}$ \\
\hline $\begin{array}{l}\text { Entzündungen im Bereich teil-/ } \\
\text { retinierter Zähne }\end{array}$ & $\begin{array}{l}\text { Arthralgien des Kiefergelenks } \\
\text { schlecht sitzende Prothesen }\end{array}$ \\
\hline Herpes Zoster & $\begin{array}{l}\text { atypische Gesichtsschmerzen/ } \\
\text {-odontalgien }\end{array}$ \\
\hline Aphthen & Glossopharyngeusneuralgie \\
\hline Lymphadenitis & \\
\hline Eagle-Syndrom & \\
\hline
\end{tabular}

gelistet. Grundlage hierfür sind neurophysiologische Zusammenhänge, wie die gemeinsame sensible Versorgung von Teilen des Ohres und der Kiefergelenke, bzw. der Kaumuskulatur. Wei- terhin werden die primären Afferenzen zentral in den gleichen Hirnstammarealen verschaltet, was bei häufigen und intensiven nozizeptiven Reizen zur Chronifizierung und Übertragungsschmerzen führen kann. Dies bedeutet, dass der Ort der Schmerzentstehung und der Ort der Schmerzempfindung nicht mehr übereinstimmen, und demnach vom Patient wie von Therapeuten fehlgedeutet werden können. Sehr häufig kann dies insbesondere bei chronischen Myalgien wie beispielsweise myofaszialen Schmerzen der Kaumuskulatur gesehen werden. Nach Travell und Simons [3], und auch nach eigenen Beobachtungen sind hier insbesondere der M. masseter pars profunda, der M. pterygoideus medialis und lateralis zu nennen. Aber auch ein über längere Zeit schmerzhafter M. sternocleidomastoideus und M. digastricus venter posterior können periaurikuläre Beschwerden bewirken.

\subsection{Tinnitus}

Ohrgeräusche werden, so sie keine eindeutig nachweisbare Ursache haben häufig mit Erkrankungen im Kieferbereich in Verbindung gebracht. Gründe hierfür sind u.a. anatomische Begebenheiten, wie eine gemeinsame Innervation der Kaumuskulatur und der Muskeln M. tensor tympani, M. tensor veli palatini und M. levator veli palatini durch den N. trigeminus. Einige Autoren

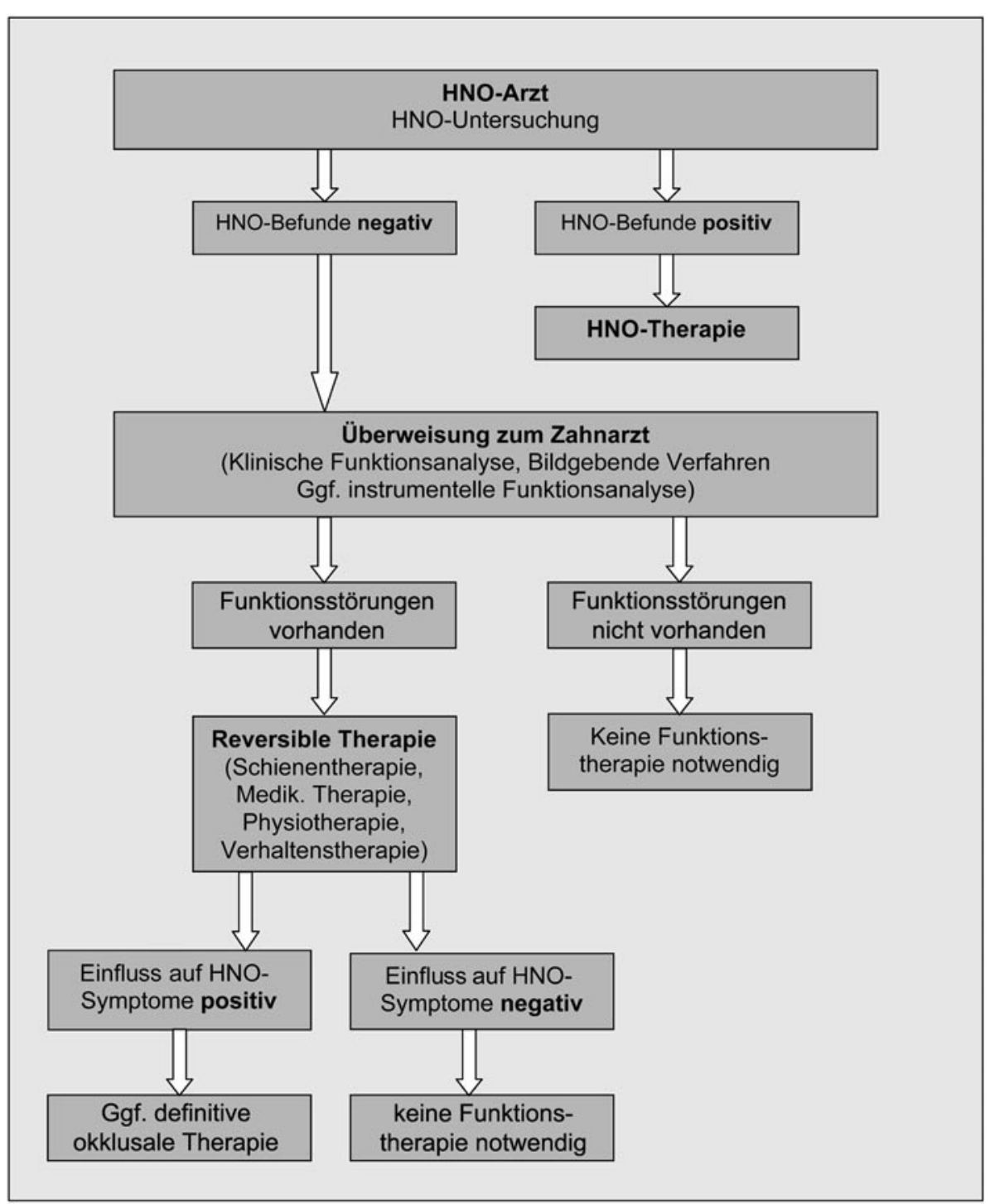

Abb. 1 Schema zum diagnostischen und therapeutischen Vorgehen bei Patienten mit Ohrenschmerzen. 
schlussfolgerten daraus Zusammenhänge, dass eine verspannte Kaumuskulatur infolgedessen Störungen wie Tinnitus und subjektiv reduziertes Hörvermögen verursachen könne. Obwohl diese Vermutungen nicht bestätigt werden konnten, bekräftigt eine neue Studie [4] die Beobachtung, dass Tinnitus-Patienten signifikant häufiger auch unter palpationsempfindlicher Kaumuskulatur und Kiefergelenken sowie schmerzhafter Mundöffnung leiden als Kontrollpatienten. Eine unmittelbare, kausale Beziehung ist dadurch jedoch nicht gegeben. Vielmehr kann vor dem Hintergrund, dass zirka 3\% der Bevölkerung unter CMD leiden [1] und Tinnitus mit einer Prävalenz von $14-32 \%$ in der Normalbevölkerung verbreitet ist, eine häufige, zufällige Koinzidenz erwartet werden. Beide Erkrankungen scheinen darüber hinaus vielfach im Zusammenhang mit körperlichen oder psychosozialen Belastungsphasen zu entstehen, bzw. verstärkt zu werden. Somit könnte auch von der Hypothese ausgegangen werden, dass eine gemeinsame Ursache wie zum Beispiel Stress unterschiedlicher Genese zwei unterschiedliche Krankheitsbilder hervorruft, die jedoch nicht in einer direkten Beziehung zueinander stehen. Während einerseits Studien existieren, die eine Verbesserung des Tinnitus nach zahnärztlicher Therapie beschreiben [5-8] wird dies in einer anderen Arbeit bestritten [9]. Die Beziehung zwischen Tinnitus und CMD ist letztlich noch wenig erforscht und bleibt vorerst kontrovers.

\subsection{Sinusitiden}

Die Ursachen schmerzhafter Erkrankungen der Kieferhöhle sind in aller Regel gut diagnostizierbar und unschwer von CMD abzugrenzen. Jedoch erscheint erwähnenswert, dass chronische Myalgien insbesondere des M. masseter pars superficialis, des M. temporalis und der Mm. pterygoidei medialis et lateralis Übertragungsschmerzen in Areale bewirken können, die der Patient auch als Kieferhöhlenentzündung beschreiben kann. Eine konsiliarisch durchgeführte, zahnärztliche Funktionsanalyse kann hier bei der Diagnosefindung hilfreich sein.

\subsection{Eagle-Syndrom}

Dieses Beschwerdebild, welches mit neuralgiformen Ohrenschmerzen, Halsschmerzen, Schluckbeschwerden, Kopfschmerzen und Nackenschmerzen, insbesondere bei einseitigen Kopfbewegungen einhergeht, beruht auf einem verlängerten Processus stylohyoideus und/oder einem verknöcherten Ligamentum stylohyoideum (Abb.2). Eine klinische Palpationsempfindlichkeit und eine Röntgenaufnahme dieses Areals können einen solchen Verdacht bestätigen. Differenzialdiagnostisch sind davon myogene Beschwerden der posterioren akzessorischen Kaumuskulatur, wie dem M. digastricus venter posterior oder der M. stylohyoideus aber auch des M. sternocleidomastoideus abzugrenzen.

\section{5 Überweisung zum Zahnarzt}

Die Notwendigkeit einer konsiliarischen Überweisung zum CMD-fachkundigen und funktionsanalytisch tätigen Zahnarzt ist immer dann gegeben, wenn sich in der HNO-ärztlichen Untersuchung keine pathologischen Befunde ergeben. Im Vordergrund steht hierbei, ob durch eine Veränderung der Unterkieferlage eine Beeinflussung der HNO-Symptome möglich ist. Ist dies der Fall, empfiehlt sich eine ausführliche klinische, gegebenenfalls auch weiterführende Funktionsanalyse. Abb. 1 zeigt mögliche Indikationen für eine Überweisung zum Zahnarzt sowie schema-

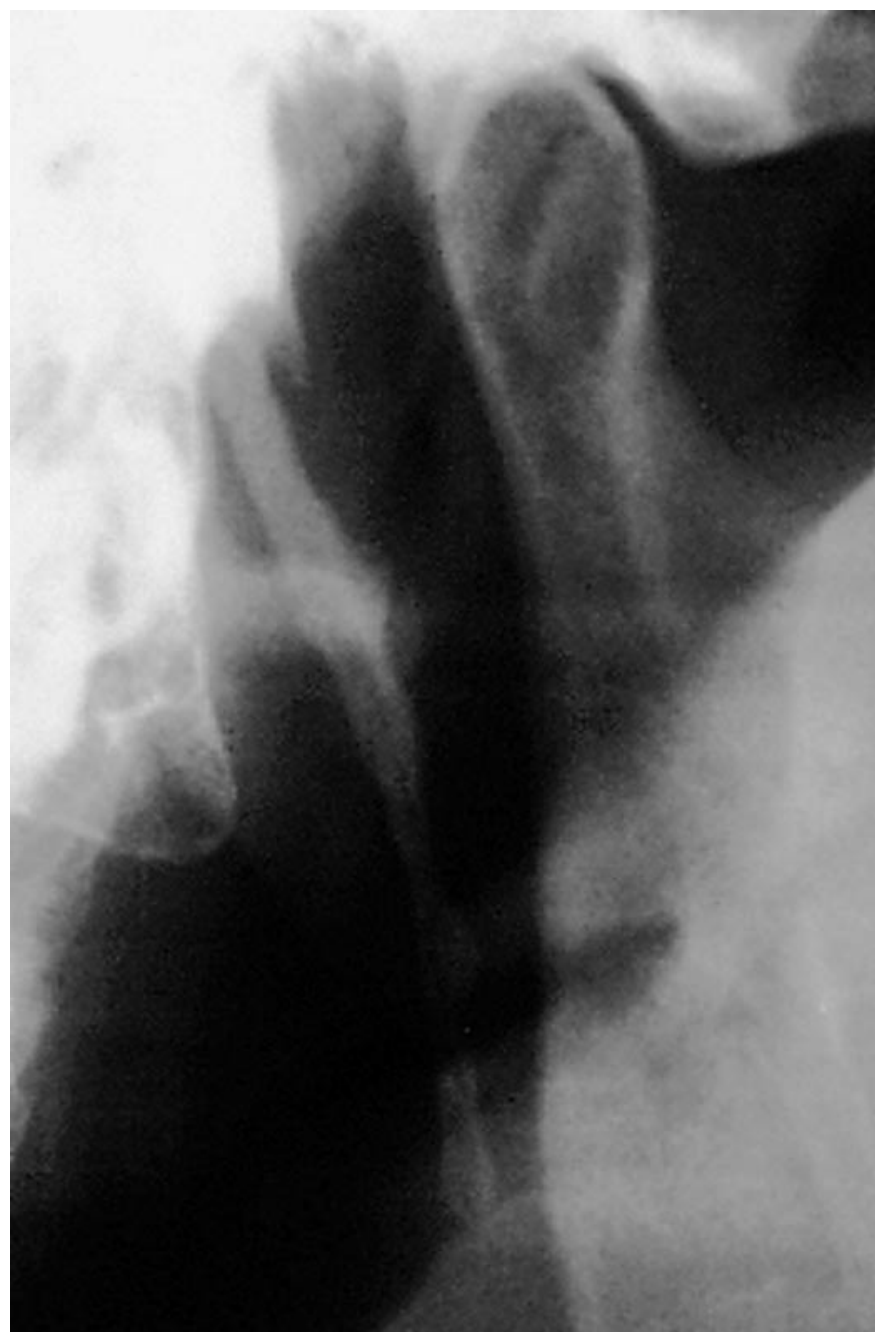

Abb. 2 Eagle-Syndrom - verlängerter Processus stylohyoideus.

tisch ein mögliches diagnostisches und therapeutisches Vorgehen bei entsprechenden Patienten auf.

\section{Diagnostik}

Die Diagnostik von CMD sollte sich, wie für alle anderen Bereiche einer präventionsorientierten Zahnmedizin, in eine Basisdiagnostik und eine erweiterte Diagnostik gliedern [10]. Darüber hinaus kommen weiterführende diagnostische Verfahren zur Anwendung, wenn Symptome und Befunde einer CMD vorliegen und diese einer näheren ursächlichen Abklärung bedürfen [11].

\subsection{Basisdiagnostik: Funktioneller Kurzbefund als CMD-Screening}

Grundsätzlich sollte bei jedem Patienten, der zahnärztlich oder ärztlich untersucht und behandelt wird, neben der Anamnese und der extra- und intraoralen Untersuchung aus medizinischen aber auch forensischen Gründen eine funktionelle Kurzuntersuchung bzgl. einer eventuell bestehenden CMD durchgeführt werden. Dafür sollte eine orientierende Untersuchung hinsichtlich einer Schmerzhaftigkeit der Muskulatur und der Kiefergelenke, einer Limitation und Deviation bei Kieferöffnung sowie instabiler bzw. gestörter Okklusionsverhältnisse erfolgen. Ergeben sich 


\section{Screening}

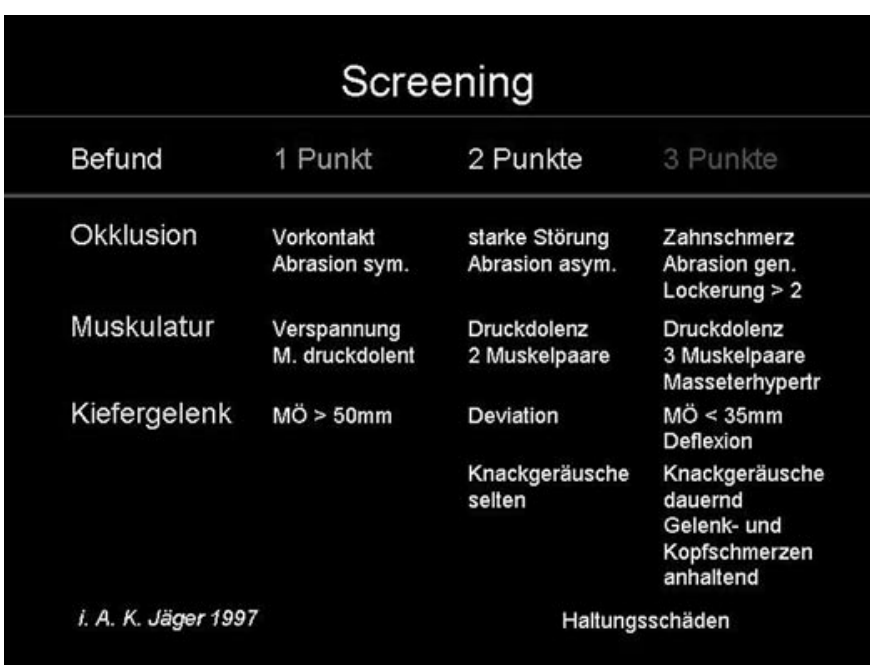

Abb. 3 Screening-Schema in Anlehnung an Jäger 1997, 1 kein Diagnose- und Therapie-Bedarf, 2 Diagnose-Bedarf, 3 Diagnose- und Therapie-Bedarf.

bei dieser Untersuchung pathologische Befunde können sie in ihrem Schweregrad in Anlehnung an Jäger (Abb. 3) [12] bewertet und eine erweiterte Diagnostik eingeleitet werden (siehe 2.2).

\subsection{Erweiterte Diagnostik: Klinische Funktionsanalyse}

Die klinische Funktionsdiagnostik dient bei Patienten mit Verdacht auf CMD der Erhebung und Dokumentation von Befunden an den Zähnen, der Muskulatur, den Kiefergelenken und angrenzenden Strukturen [13 - 15]. Um HNO-Ärzten einen Überblick zu geben, wie die erweiterte zahnärztliche Diagnostik abläuft, wird diese nachfolgend in ihren wesentlichen Elementen beschrieben. Die eigentliche Durchführung der Untersuchung ist dem Zahnarzt in gewissem Maße freigestellt. Es existieren aber verschiedene, in Nuancen voneinander abweichende Vorschläge $[16,18,19]$. Als Orientierung dafür mag auch der Untersuchungsbogen der Arbeitsgemeinschaft für Funktionsdiagnostik und Therapie in der DGZMK (www.dgzmk.de) gelten, der 1985 erstmals publiziert und jüngst von Reiber und Ottl [17] aktualisiert wurde. Notwendig ist in jedem Fall die Dokumentation der Befunde in einem geeigneten Formblatt $[18,19]$.

\subsubsection{Anamnese}

$\mathrm{Zu}$ Beginn der Anamnese sollten Fragen nach genauer Lokalisation, Beginn und Auslöser aller Hauptbeschwerden im Mittelpunkt stehen (wo, wie, wann, wie oft, warum). Hierzu gehören Fragen nach der Schmerzqualität, der Häufigkeit und Dauer der Problematik und eventuell bestehender Begleiterscheinungen. Weiterhin sollte nach Schwankungen oder Spontanremissionen gefragt werden, wozu auch Fragen nach verstärkenden und lindernden Faktoren gehören. Um bei bestehenden Schmerzen einen Eindruck von der Schmerzintensität zu bekommen, unter der ein Patient leidet, empfiehlt es sich, Schmerzskalen (z. B. Visuelle Analog Skala, VAS) zu verwenden, mit der der Patient seine aktuelle und durchschnittliche Schmerzintensität beschreiben kann. Einen wichtigen Teil der Anamnese stellt die Frage nach allgemeinmedizinischen Erkrankungen dar. Insbesondere Erkrankungen in angrenzenden Bereichen wie Sinus- und Ohrerkrankungen, aber auch Erkrankungen des rheumatischen Formenkreises und orthopädische Probleme müssen aufgrund ihrer offensichtlichen Einflussmöglichkeiten auf craniomandibuläre Dysfunktionen erfragt werden. Jedoch müssen auch Erkrankungen des Herz-Kreislauf-Systems, des Magen-Darm-Systems, Allergien sowie Störungen der Leber und Nierenfunktion bekannt sein. Eingenommene Medikamente müssen beachtet werden, da diese eine heute wieder wichtiger werdende medikamentöse Therapie zum Teil erheblich beeinflussen können. Traumata, Tumoren im Kopf-, Kiefer-, Gesichtsbereich und Nackenverletzungen können ursächliche Faktoren für CMD sein und müssen erfragt werden. Die Anamnese eines Patienten mit Verdacht auf CMD beinhaltet selbstverständlich auch Fragen nach Veränderungen des Zahnersatzes und der Okklusion, die im zeitlichen Zusammenhang mit der Entstehung der Hauptbeschwerden stehen, sowie nach Parafunktionen oder anderen oralen Habits. Während die oben angeführten Punkte im Wesentlichen selbstverständlich sind, wird die psychosoziale Anamnese meist vernachlässigt oder sogar gänzlich außer Acht gelassen. Heute bestehen allerdings kaum mehr Zweifel, dass Depression, Ängste und andere psychische Faktoren, aber auch Probleme am Arbeitsplatz, in der Familie, finanzielle Sorgen, laufende Gerichtsverfahren etc. einen erheblichen Einfluss sowohl auf die Entstehung als auch für die Therapie von CMD haben.

\subsubsection{Schmerzlokalisation}

Zeichnungen zur Schmerzlokalisation, wie Kopf- und Ganzkörperschemata, können eine wertvolle diagnostische Hilfe sein, um Probleme am Kopf und in anderen Körperbereichen, wie etwa Projektions- und Übertragungsschmerzen, multiple Arthritiden, Probleme in der Hals-, Brust- und Lendenwirbelsäule und des Beckens, darzustellen. Zeichnungen der Kopf- und Halsregion gehören daher seit Anfang der 60er-Jahre regelmäßig zur erweiterten Anamnese im Rahmen der klinischen Funktionsanalyse (Abb. 4).

\subsubsection{Palpation}

Vor der eigentlichen Untersuchung sollten Asymmetrien, die Haltung des Patienten, der Gesichtsausdruck, Schwellungen u. Ä. inspiziert werden. Der obligatorische Palpationsbefund der eigentlichen (Abb.5) sowie der akzessorischen Kaumuskulatur (Abb.6) und der Kiefergelenke (Abb. 7) ist ein wesentlicher Grundstein für die Beurteilung des funktionellen Zustandes craniomandibulärer Strukturen. Der Verspannungsgrad und die Schmerzhaftigkeit geben Hinweise auf eine CMD-Symptomatik. Die Palpation der Muskulatur stellt eine schwierige Untersuchung dar, die vom Zahnarzt wie vom Patienten eine gute Kooperation wie auch in doppelter Hinsicht ein „sich Einfühlen“ verlangt - Eigenschaften, die vom (Zahn-) Arzt regelmäßig verlangt werden, die aber häufig vernachlässigt werden. Auch Kiefergelenkgeräusche, wie Knacken oder Reiben, können palpiert oder auskultiert werden. Es sei jedoch schon an dieser Stelle vorweggenommen, dass ein Knacken in einem oder beiden Kiefergelenken zwar ein häufiger Befund in der Gesamtbevölkerung ist aber für sich genommen, wenn keine weiteren zahnärztlichen Maßnahmen, wie eine kieferorthopädische Behandlung oder prothetische Rekonstruktionen folgen und wenn das psychosoziale Befinden nicht eingeschränkt ist, keine Indikation für eine Therapie darstellt. Neben dem obligatorischen Zahnbefund sollte auch eine parodontale Kurzuntersuchung erfolgen (PAR-Kurzbefund oder Periodontal Screening Index [20]). Unabdingbar für eine Differenzialdiagnose ist zudem ein Befund der Weichgewebe, 

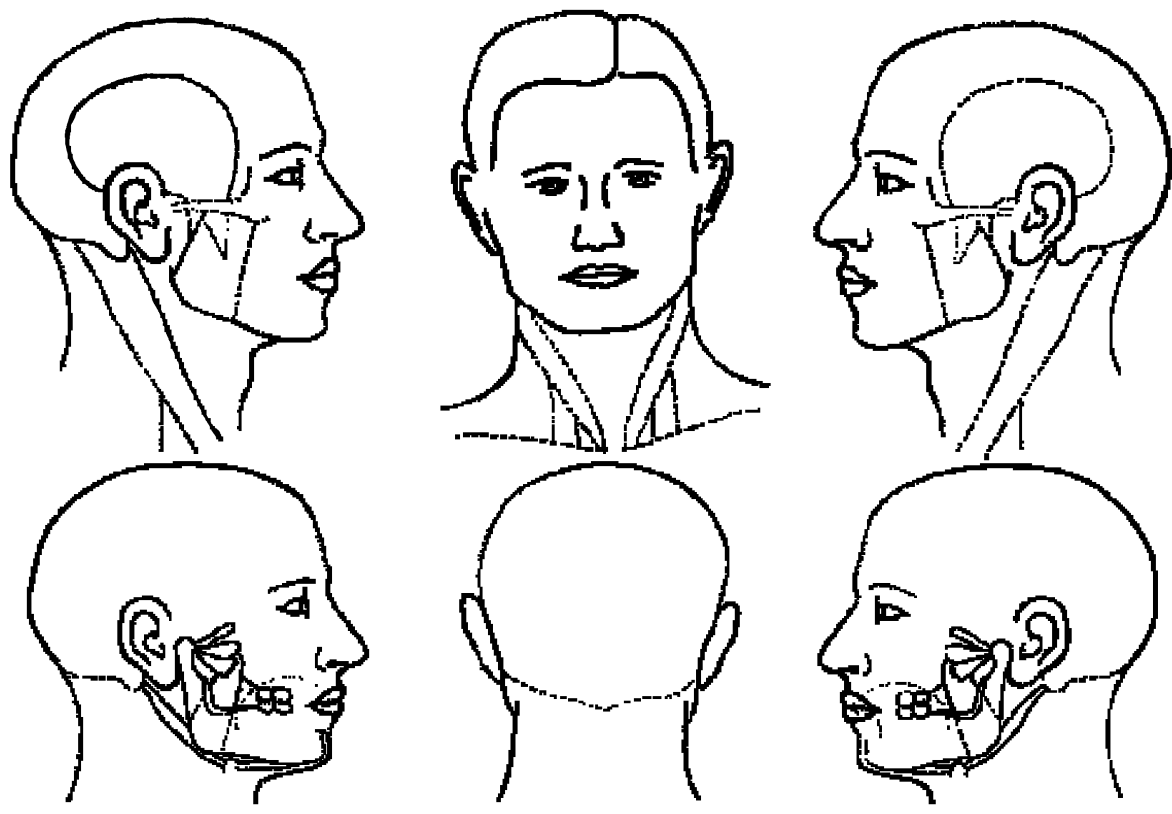

Abb. 4 „Krogh-Poulsen“-Köpfe zur Einzeichnung der Schmerzlokalisation, der Schmerzausbreitung und der Druckempfindlichkeit. um beispielsweise Schleimhautveränderungen und Tumoren jener Gewebe als Schmerzursache auszuschließen.

\subsubsection{Okklusionsdiagnostik}

Auch wenn der Okklusion in der Entstehung und Aufrechterhaltung von CMD heute nicht mehr der herausragende Stellenwert beigemessen wird wie noch vor einigen Jahren, so besitzt der Okklusionsbefund hinsichtlich der dentalen, orthopädischen Stabilität in der klinischen Untersuchung eine zentrale Rolle [21,22]. Bestimmten Malokklusionen scheint eine besondere Bedeutung hinsichtlich CMD zuzukommen. Hierzu gehören ein frontal offener Biss (Abb. 8), ein Overjet größer als 6 mm (Abb. 9), aber auch eine Übereinstimmung von retraler und habitueller Kontaktposition, da sich hierbei das Gelenk in einer Grenzposition befindet. Das Fehlen von mehr als vier Zähnen im Seitenzahngebiet (posteriorer Stützzonenverlust) scheint ebenfalls zu CMD führen zu können $[23,24]$. Durch eine klinische Okklusionsanalyse können zum Beispiel vorzeitige Kontakte erkannt werden, die Ursache für ein dysfunktionelles Geschehen sein können. Weiterhin sollten Veränderungen an den Zähnen und dem Parodontium, wie exzessive Schliffflächen oder Rezessionen, die mit parafunktionellen Bewegungen und Belastungen in Verbindung gebracht werden, erhoben werden. Dadurch ist es oft möglich, Kofaktoren einer CMD zu erkennen und entsprechende therapeutische Schritte einzuleiten. Wichtig ist allerdings an dieser Stelle zu erwähnen, dass die Autoren bei den vorgenannten Befunden lediglich ein gehäuftes gemeinsames Vorkommen mit CMD fanden aber keine kausale Beziehung herstellen konnten.

\subsubsection{Neurologische Kurzuntersuchung}

Definitionsgemäß zählen nur Erkrankungen der Kaumuskulatur, der Kiefergelenke und angrenzender Strukturen zu CMD. Einige Patienten berichten jedoch auch hierbei über Symptome wie Taubheitsgefühl oder Allodynie. Um einen Anhalt zu bekommen, ob ein neurologisches Geschehen an der Symptomatik beteiligt sein könnte, sollte deswegen vom Zahnarzt eine neurologische Kurzuntersuchung der Hirnnerven durchgeführt werden (Abb.10). Wenn bei diesen kurzen Untersuchungen Auffälligkei- ten erkannt werden, ist es oft notwendig, den Patienten vor einer weiteren zahnärztlichen Behandlung einem Neurologen und/ oder einem HNO-Arzt zur Ausschlussdiagnostik vorzustellen.

\subsubsection{Orthopädische Kurzuntersuchung}

Es besteht heute kein Zweifel mehr, dass Wechselbeziehungen zwischen dem HWS-Bereich und dem orofazialen System bestehen. Deswegen und aufgrund der großen Zahl von Patienten, die gleichzeitig Probleme im HWS-Bereich und im orofazialen System aufweisen, sollte ähnlich der neurologischen Kurzuntersuchung auch ein orthopädisches Screening der HWS-, Nackenund Schulterregion durchgeführt werden [11,25]. Dieses sollte mindestens eine Überprüfung der Kopfbeweglichkeit (Abb.11) und damit einhergehender Schmerzen sowie die Palpationsempfindlichkeit der Nackenmuskulatur (Abb. 12,13) umfassen. Ergeben sich hier Auffälligkeiten, sollte der Patient einer weiterführenden Untersuchung durch einen Orthopäden und/oder einem Physiotherapeuten zugeführt werden.

\subsection{Weiterführende Diagnostik}

$\mathrm{Zu}$ den weiterführenden diagnostischen Maßnahmen in der Funktionsdiagnostik zählen: psychometrische Tests (z.B. SCL-90R, Brief Symptom Inventory BSI $[26,27])$, die Erfassung von Intensität und Periodizität von Beschwerden und Schmerzen, die klinische Strukturanalyse, instrumentelle Analyseverfahren (Okklusionsanalyse und Bewegungsanalyse) und bildgebende Verfahren (MRT, seltener CT, Sonographien und Szintigraphien).

\subsubsection{Manualmedizinische Strukturanalyse}

Die manualmedizinische („manuelle“) Strukturanalyse [28] beruht auf anerkannten orthopädischen Verfahren, der manuellen und isometrischen Testung von artikulären und myogenen Gewebsstrukturen hinsichtlich ihrer Belastbarkeit, Bewegungsmöglichkeit und Empfindlichkeit. Mit diesen Tests (auch als „Provokationstest“ oder als „manuelle Tests“ bezeichnet) ist es möglich, pathologische Veränderungen in den Gelenken oder der Muskulatur zu erkennen (Abb. 14). 


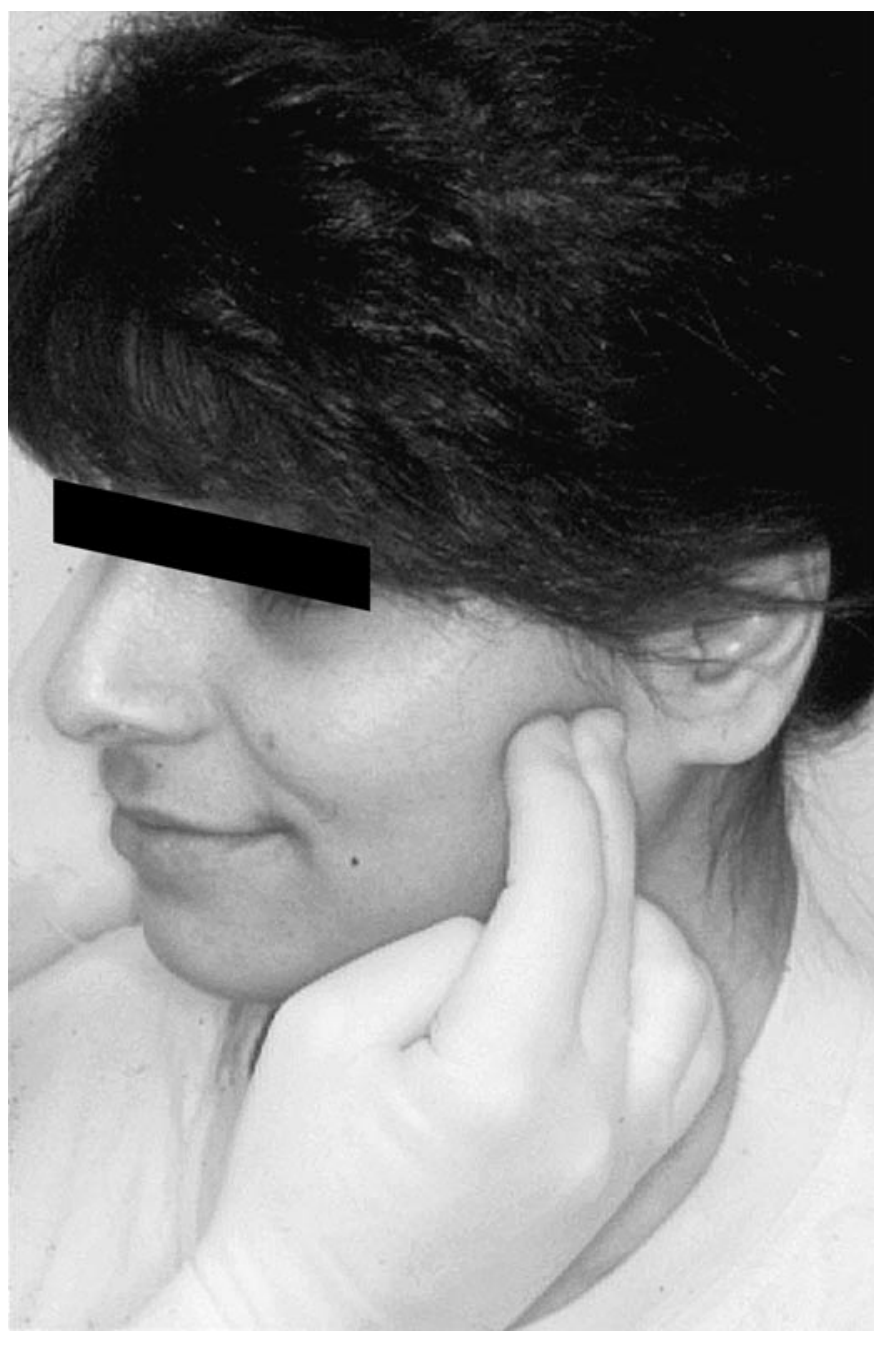

Abb. 5

\subsubsection{Instrumentelle Diagnostik}

Die instrumentelle Diagnostik gliedert sich in die Verfahren der instrumentellen Okklusionsdiagnostik, der instrumentellen Aufzeichnung der Kondylenposition und der Aufzeichnung der Kiefergelenkbewegung [25]. Diese Verfahren sind dann anzuwenden, wenn aus der klinischen Diagnostik keine eindeutige Diagnose erhalten wurde bzw. vorliegende klinische Befunde genauer verifiziert werden sollen. Die Okklusionsdiagnostik in einem Artikulator dient der Erkennung von Störungen in statischer und dynamischer Okklusion. Eine eindeutige Bestimmung des okklusalen Zustandes ist jedoch nur dann möglich, wenn keine Gelenkpathologie vorliegt. Eine instrumentelle Bewegungsaufzeichnung (Axiographie, Pantographie) mit mechanischen oder elektronischen Geräten ist dann indiziert, wenn klinisch vorliegende Bewegungsstörungen nicht eindeutig einer Gelenkpathologie (Diskusverlagerung, Strukturveränderung) zugeordnet werden können. Aus den Aufzeichnungsspuren kann indirekt auf zugrunde liegende Krankheitsbilder geschlossen werden. Darüber hinaus dient die instrumentelle Bewegungsaufzeichnung dazu, metrische Daten für die Justierung eines Artikulators (Kausimulators) zu erhalten.

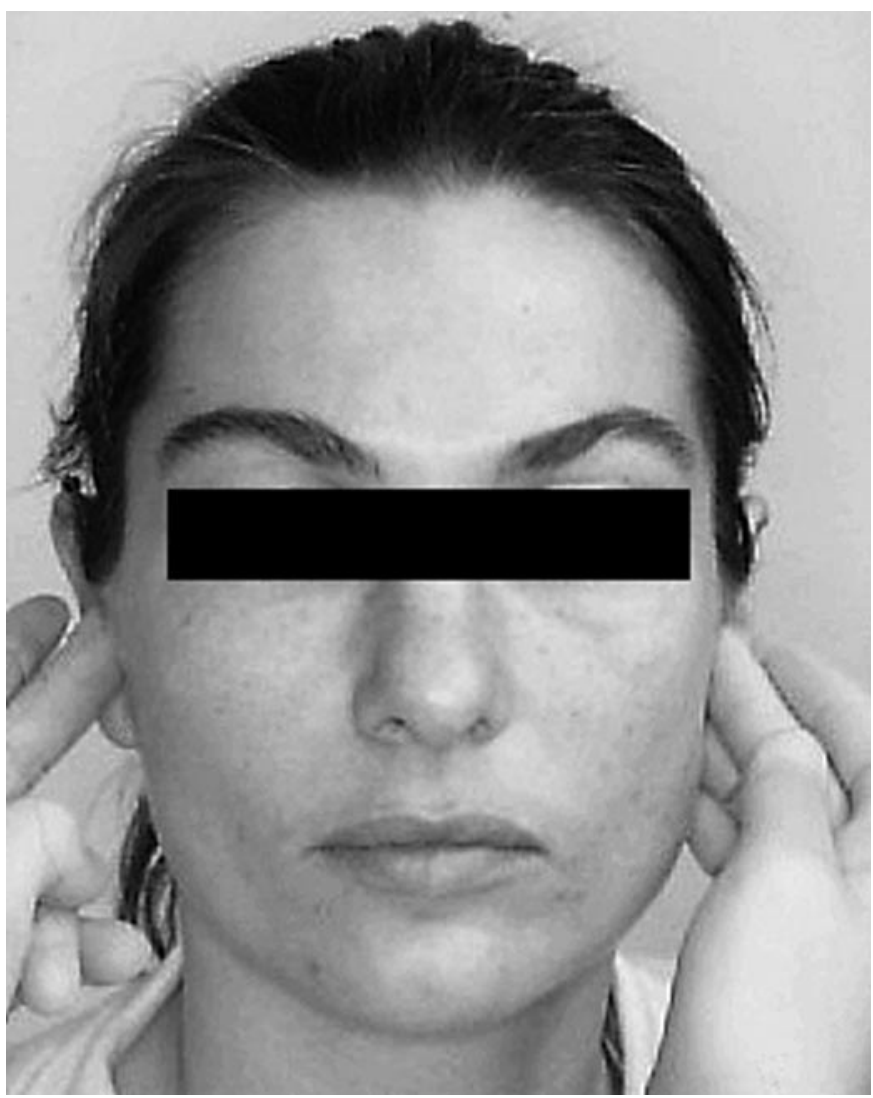

Abb. 6

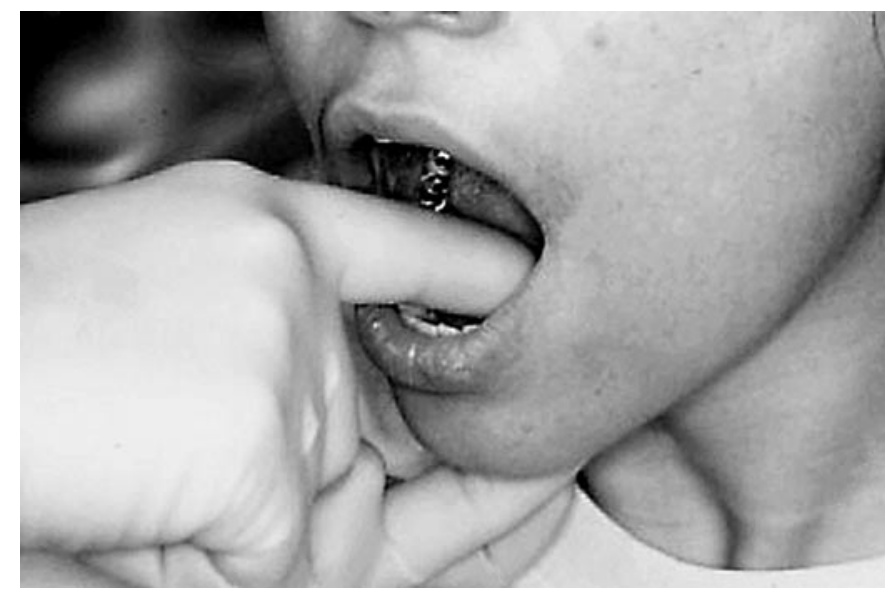

Abb. 5-7 Palpation des M. masseters, der Kiefergelenke und des Mundbodens.

\subsubsection{Bildgebende Diagnostik}

Die bildgebende Diagnostik hat sich in den letzten 20 Jahren grundsätzlich verändert. Stand vor dieser Zeit nur die Diagnostik mittels schräglateralem Röntgenbild (SLR) für die Kiefergelenkdarstellung zur Verfügung, wurde diese durch die Entwicklung der Computertomographie (CT) (Abb. 15) und Kernspintomographie (MRT) (Abb. 16) weitgehend verdrängt. Auch wenn diese Verfahren teurer sind als ein SLR, so geben sie doch mehr und detailliertere Informationen über pathologische Veränderungen an den Kiefergelenken [29]. Das CT ist indiziert, wenn aus der klinischen Analyse der Verdacht auf eine strukturelle Veränderung an den Gelenkflächen besteht. Auch ist eine Stellungsdiagnostik mit 


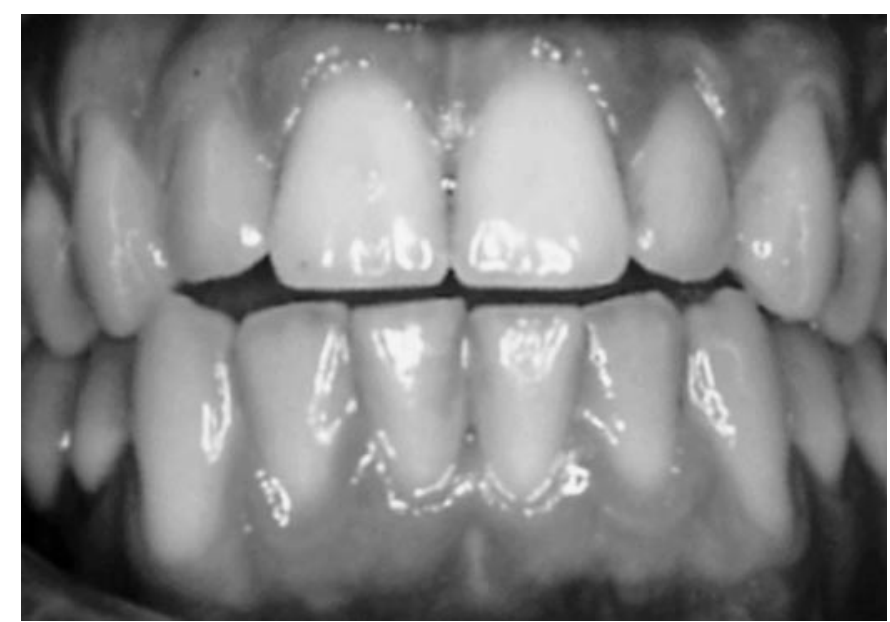

Abb. 8 Frontal offener Biss.

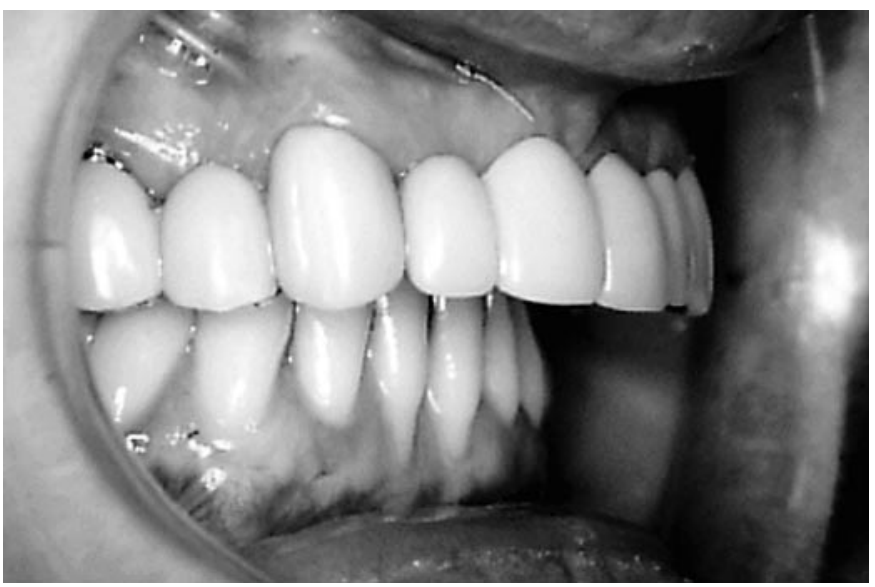

Abb. 9 Patientin mit einem ausgeprägten horizontalen Überbiss.
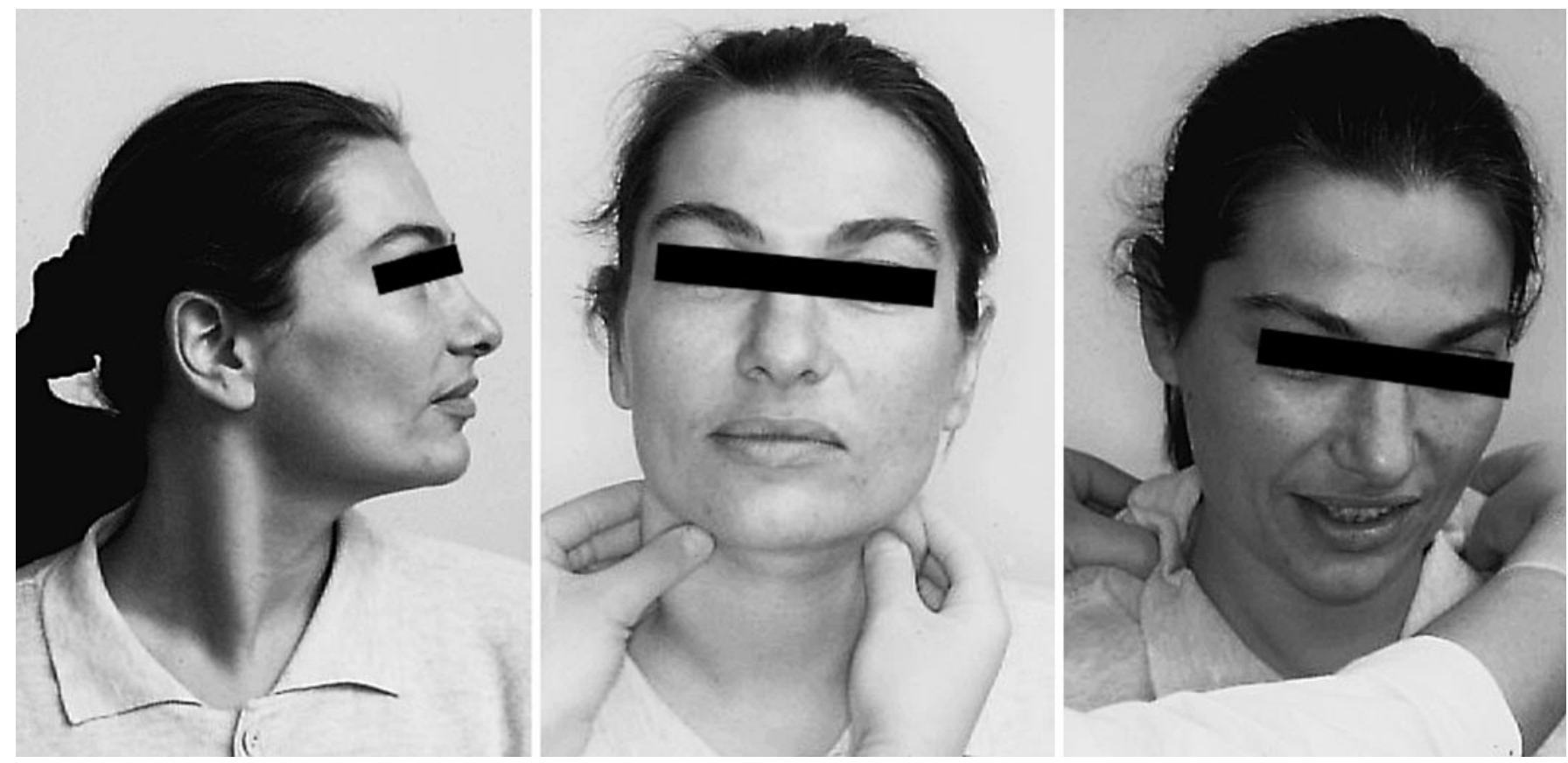

Abb. 11 - 13 Überprüfen der Beweglichkeit der Kopfgelenke und der Halswirbelsäule; Palpation des M. sternocleidomastoideus.

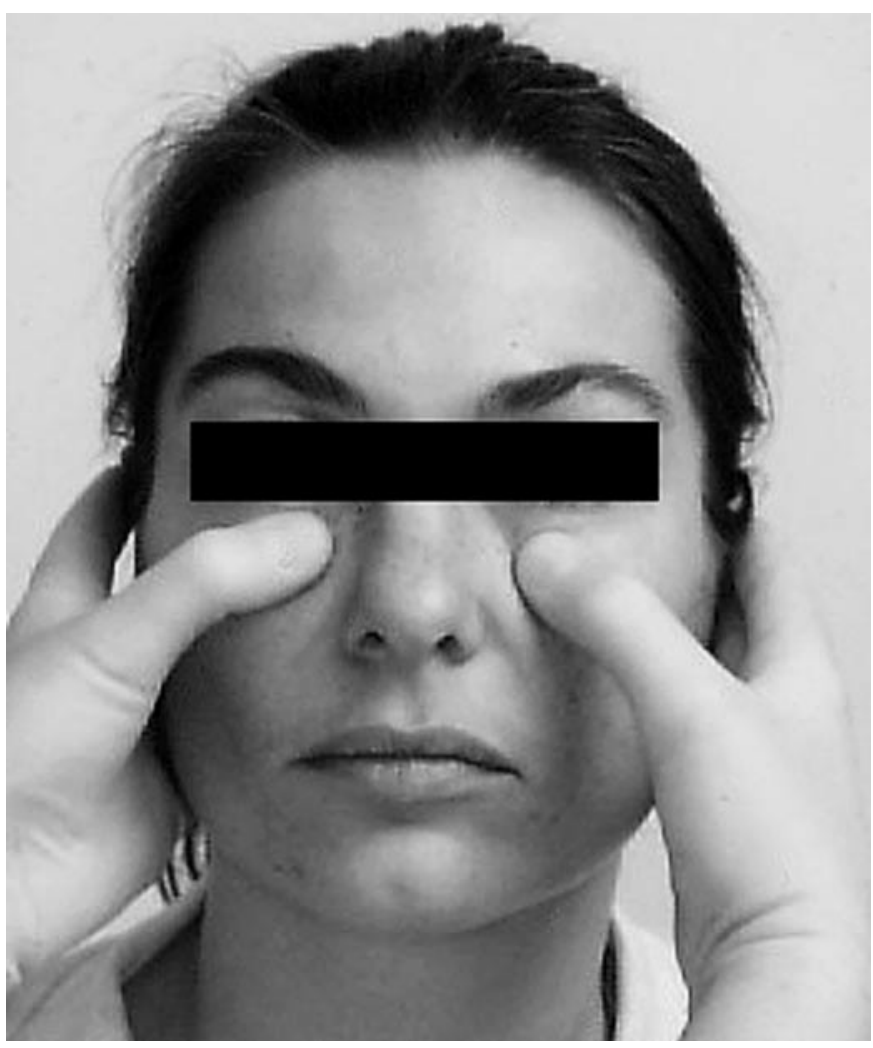

Abb. 10 Palpation der Nervenaustrittspunkte des 2. Trigeminusastes. 


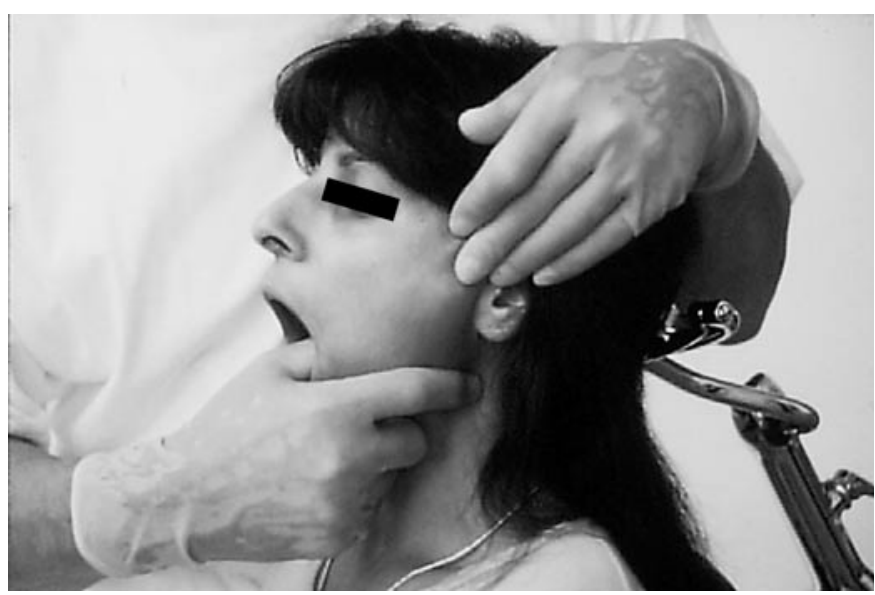

Abb. 14 Manueller Provokationstest des linken Kiefergelenks auf Traktion.

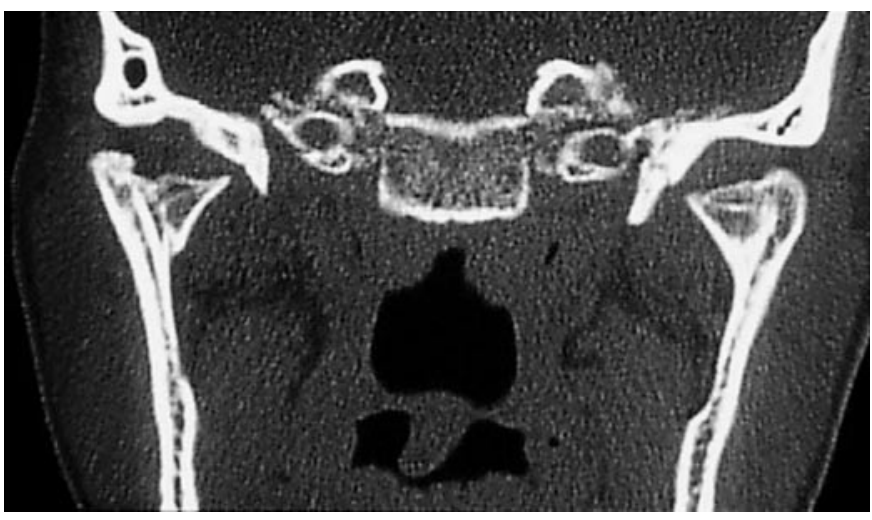

Abb. 15 Computertomogramm bei beidseitiger Osteoarthrose nach Kollumfraktur.

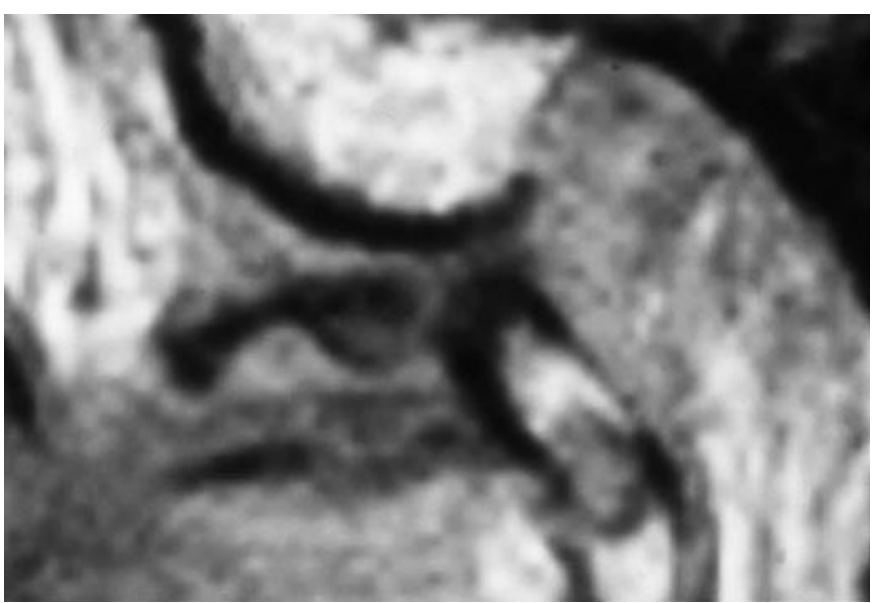

Abb. 16 Kernspintomogramm eines Kiefergelenkes: anteriore Diskusverlagerung ohne Reposition und strukturelle Veränderung im posterioren Bereich des Diskus.

dem in axialer Schichtung angefertigten CT möglich. Darüber hinaus können weitere knöcherne und raumfordernde Veränderungen wie z. B. Tumoren erkannt werden.

Das MRT [29] kommt vor allem zur Anwendung, wenn nach der erweiterten Diagnostik der Verdacht auf eine Diskusverlagerung besteht, die aber in Art und Stellung klinisch nicht eindeutig ve- rifiziert werden kann oder der Verdacht auf einen raumfordernden Prozess besteht. Die Aufnahmen sollten bei geschlossenen Zahnreihen und bei weit geöffnetem Kiefer in $\mathrm{T}_{1}$-Wichtung angefertigt werden. Auf diesen Aufnahmen ist in verschiedenen Schichtebenen die Lage des Discus articularis zum Kondylus meist eindeutig zu erkennen. Ergänzend können mit einem MRT in $T_{2}$-Wichtung Entzündungen in den verschiedenen Arealen des Kiefergelenks dargestellt werden.

\subsection{Auswertung: Diagnoseschema}

Grundsätzlich sollten alle erhobenen anamnestischen Informationen und Befunde in eine eindeutige Diagnose münden. Historisch sind hierfür verschiedene zusammenfassende Diagnosen eingeführt worden, u.a. orofaziale Funktionsstörung, orofaziales Schmerzsyndrom, myofasziales Schmerzsyndrom, mandibuläres Dysfunktionssyndrom $[15,30]$. In Deutschland besondere Verbreitung gefunden hat der von Schulte angegebene Begriff „Myoarthropathie“ [31]. Schulte selbst erkannte allerdings schon wenige Jahre später, dass diese Bezeichnung der komplexen Pathogenese des Krankheitsbildes nicht gerecht wurde und erweiterte den Begriff zur „Myo-Arthro-Okkluso-Neuro-Psychopathie“ [32]. Als Weiterentwicklung basierend auf dem Diagnoseschema von Schulte (1980) und Freesmeyer (1993) [25] stellte eine Gruppe von Wissenschaftlern deutschsprachiger Universitäten 2001 ein neues Diagnoseschema vor, was auf einer Einteilung in Initialbzw. Haupt-, Neben- und Differenzialdiagnosen beruht [33].

- Die Diagnose(n) (Tab.2) ergibt oder ergeben sich aus den anamnestischen Informationen, der Basisdiagnostik und der erweiterten Diagnostik, die im Rahmen der klinischen Funktionsanalyse erhoben wurden.

- Bei aktuell erweitertem Untersuchungsumfang der zahnärztlichen klinischen Funktionsanalyse erlaubt diese auch Rückschlüsse auf die Nebendiagnosen (Tab. 3), welche die diagnostische Unterteilung der Globaldiagnose CMD ergänzen.

- Differenzialdiagnosen (Tab.3) ergeben sich auch aus der zahnärztlichen erweiterten und weiterführenden Diagnostik, bedürfen aber meist einer interdisziplinären Konsultation und Therapie und schließen bei ihrer Bestätigung in der Regel die Initialdiagnose aus.

Alternativ hierzu sind internationale Klassifikationen zu nennen, wie beispielsweise die International Classification of Diseases (ICD-10) [34] oder die in Zusammenarbeit mit der American Academy of Orofacial Pain entstandenen Untergruppen 11.7 und 11.8 der Klassifikation der International Headache Society (IHS) $[35,36]$.

\section{$4 \quad$ Therapie}

Wenn CMD-Patienten mit Beteiligung von Faktoren aus anderen Fachgebieten aus rein zahnärztlicher Sicht behandelt werden und allgemeinmedizinisch Aspekte nicht beachtet werden, kann man davon ausgehen, dass Patienten nicht erfolgreich behandelt werden. Neben Patienten, bei denen die Funktionseinbuße im Vordergrund steht, gilt dies insbesondere für Patienten mit chronischen Schmerzen als Spätfolge einer zuvor nicht oder nicht erfolgreich behandelten CMD. Deshalb ist die interdisziplinäre Kommunikation Grundlage für einen Behandlungserfolg. Die Therapie von CMD gliedert sich in folgende Schritte, die sich im- 
Tab. 2 Gliederung der Diagnosen in drei Hauptgruppen und deren typische Ausprägungen

\begin{tabular}{|c|c|}
\hline \multicolumn{2}{|l|}{ Initialdiagnosen } \\
\hline Okklusopathie & $\begin{array}{l}\text { Veränderungen an Zähnen und Parodontien, wie Attritio- } \\
\text { nen, Abrasionen, keilförmige Defekte, nichtentzündliche } \\
\text { Gingivaretraktionen, Zahnlockerungen und okklusale } \\
\text { Traumata, die auf eine gestörte statische und/oder ge- } \\
\text { störte dynamische Okklusion und/oder die (an das Vor- } \\
\text { handensein von Okklusion gebundenen) Parafunktionen } \\
\text { Pressen und Knirschen zurückzuführen sind. }\end{array}$ \\
\hline Myopathie & $\begin{array}{l}\text { Veränderungen an der Kaumuskulatur, wie Verspannun- } \\
\text { gen, Druckempfindlichkeit oder Schmerzen der Muskel- } \\
\text { gruppen (Elevatoren, Depressoren, Protraktoren oder } \\
\text { Retraktoren), die auf Fehlfunktion zurückzuführen sind. }\end{array}$ \\
\hline Arthropathie & $\begin{array}{l}\text { Veränderungen am oder an den Kiefergelenk/en, wie } \\
\text { Verlagerungen gegenüber der zentrischen Position in } \\
\text { kranialer Richtung (Kiefergelenkkompression), in kauda- } \\
\text { ler Richtung (Kiefergelenkdistraktion), in retraler Rich- } \\
\text { tung (Retrallage), ventraler Richtung (Doppelbiss; } \\
\text { Sunday bite), Strukturveränderungen am Diskus (Diskus- } \\
\text { perforation) am Kondylus (Osteoarthrose aktiv oder } \\
\text { inaktiv), die auf Fehlfunktionen zurückzuführen sind. } \\
\text { Darüber hinaus Diskopathien, wie anterior mediale Ver- } \\
\text { lagerungen mit und ohne Reposition in der Position, die } \\
\text { der Kondylus bei habitueller Okklusion einnimmt } \\
\text { (anteriore Diskusverlagerung) oder die Verlagerung des } \\
\text { Diskus bei exkursiven Bewegungen nach posterior } \\
\text { (posteriore Diskusverlagerung). }\end{array}$ \\
\hline
\end{tabular}

Tab. 3 Neben- und Differenzialdiagnosen

\begin{tabular}{|c|c|}
\hline Craniopathie & $\begin{array}{l}\text { Veränderungen in den skeletalen und muskulären } \\
\text { Strukturen des kranialen und vertebralen Systems, die } \\
\text { auf Fehlfunktionen, Fehlhaltungen und Fehlsteuerungen } \\
\text { zurückzuführen sind. }\end{array}$ \\
\hline Fehlhaltungen & $\begin{array}{l}\text { Veränderungen in der Körperhaltung aus lateraler (Kopf, } \\
\text { Hals, Lende, Ferse) und frontaler (Kopf, Schulter, Becken) } \\
\text { Ansicht. }\end{array}$ \\
\hline $\begin{array}{l}\text { Fehlfunktionen } \\
\text { (Wirbelsäule) }\end{array}$ & $\begin{array}{l}\text { Veränderungen in der Funktion der Wirbelsäule (HWS, } \\
\text { BWS, LWS, ISG), die zur Bewegungseinschränkung } \\
\text { (Blockierungen) führen. }\end{array}$ \\
\hline $\begin{array}{l}\text { Psychosomatische } \\
\text { Faktoren }\end{array}$ & $\begin{array}{l}\text { Veränderungen im Verhalten, die auf Überlastungen } \\
\text { (Stress) physischer oder psychischer Art zurückzuführen } \\
\text { sind. Darüber hinaus zentrale psychische Veränderungen, } \\
\text { wie Depressionen, Münchhausen-S., Borderline-S., } \\
\text { Koryphäenkiller-S., Psychosen, die mit akuten und } \\
\text { chronischen Schmerzen verbunden sind. }\end{array}$ \\
\hline Neuropathien & $\begin{array}{l}\text { Veränderungen im zentralen und peripheren Nerven- } \\
\text { system, die zu typischen Schmerzreaktionen wie } \\
\text { migränoiden oder neuralgiformen Schmerzen führen. } \\
\text { Dabei kann in episodische, anfallsartige Neuropathien } \\
\text { (Bsp: Trigeminusneuralgie) und kontinuierliche Neuro- } \\
\text { pathien (Bsp: chronische, posttraumatische neuro- } \\
\text { pathische Schmerzen, postherpetische Neuralgie) } \\
\text { unterschieden werden. }\end{array}$ \\
\hline
\end{tabular}

mer auf die zugrundeliegende Diagnose beziehen: Aufklärung des Patienten, Selbstbeobachtung, Entspannungstherapie, Verhaltenstherapie, Physiotherapie, medikamentöse Therapie, therapeutische Lokalanästhesien, Schienentherapie, Stabilisierungstherapie [37], die nachfolgend in ihren Grundzügen kurz aufgezeigt werden sollen.

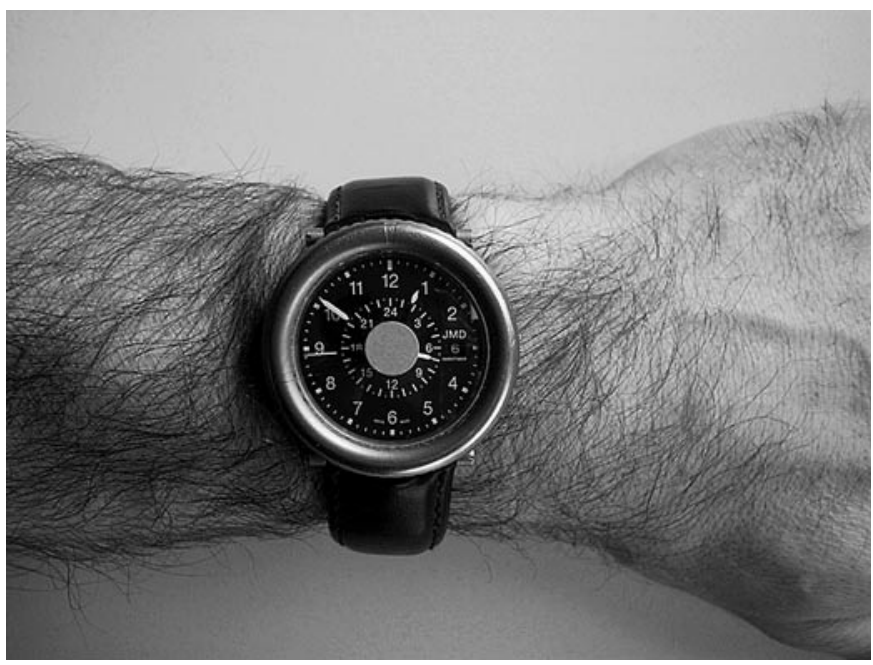

Abb. 17 Roter Punkt angebracht auf einer Uhr zur Verbesserung der Wahrnehmung von Parafunktionen.

\subsection{Aufklärung}

Der Patient ist immer über mögliche Ursachen und Zusammenhänge in der Entstehung einer CMD aufzuklären, damit er ein Verständnis für seine Symptome, Zusammenhänge bzw. Erkrankung entwickelt und letztendlich, z.B. über Selbstbeobachtung, selbst Einfluss nehmen kann. Durch die Aufklärung können dem Patienten auch Ängste bezüglich der Erkrankung oder auch der Prognose genommen werden, was häufig bereits einen positiven Einfluss auf das Beschwerdebild hat.

\subsection{Selbstbeobachtung}

Die Selbstbeobachtung [31], „was mache ich mit den Zähnen“, ist Patienten zu empfehlen, die unter Bruxomanie (Pressen und Knirschen am Tage) und z.B. an einer Masseterhypertrophie leiden. Durch Anbringen von Markern („Rote-Punkt-Technik“) am Arbeitsplatz, am Lenkrad oder anderen Orten größerer Anspannung oder Konzentration soll der Patient im ersten Schritt erkennen, ob Parafunktionen vorliegen, und wenn ja, wann diese passieren (Abb. 17). Im zweiten Schritt soll er dann erinnert werden, „was mache ich mit den Zähnen“, um aktiv durch Entspannung der Muskulatur die Fehlfunktion zu beeinflussen.

\subsection{Entspannungstechniken}

CMD werden häufig auch auf eine hohe Aktivität der Muskulatur durch Pressen und Knirschen zurückgeführt, die durch physischen und psychischen Stress noch verstärkt werden [38,39]. So sind alle Therapiemöglichkeiten wie autogenes Training, progressive Muskelrelaxation, Hypnose u.a., die zu einer muskulären Entspannung beitragen, anwendbar und dem Patienten zu empfehlen, da im Bereich des Verhaltens nur mit diesen Verfahren auch nächtlicher Bruxismus beeinflussbar ist.

\subsection{Stressmanagement, Verhaltenstherapie}

Reichen die oben beschriebenen Entspannungsverfahren nicht aus, sind mitunter tiefer greifende Verhaltensänderungen beim jeweiligen Patienten erforderlich. Hier ist in jedem Fall die konsiliarische Einbeziehung erfahrener Psychologen, Psychiater oder Ärzte für psychosomatische Medizin erforderlich [10]. 


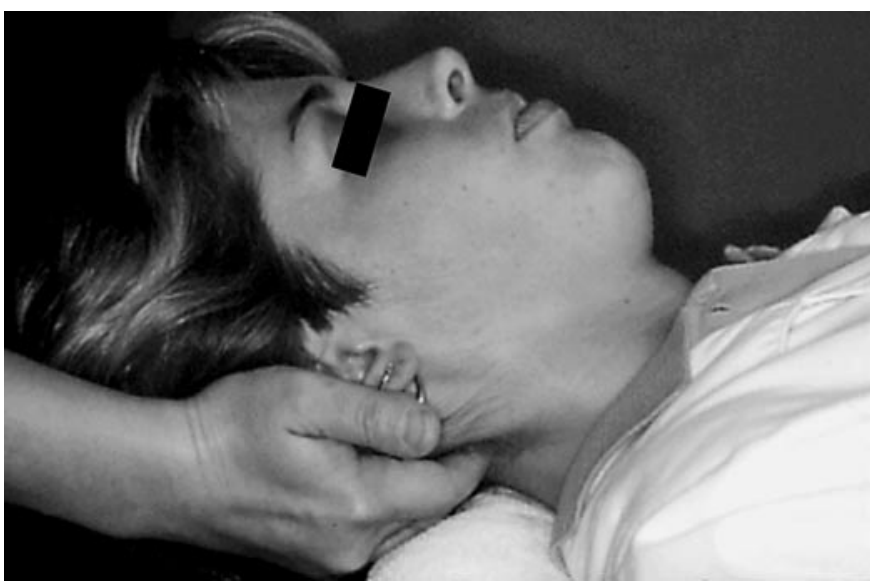

Abb. 18 Physiotherapeutische Behandlung.

\subsection{Physiotherapie}

Grundsätzlich sind funktionelle Symptome und Erkrankungen der Kaumuskulatur und der Kiefergelenke in gleicher Weise durch physikalische und physiotherapeutische Maßnahmen (Kälte, Wärme, Massagen und Bewegungsübungen) wie andere Muskeln und Gelenke des Körpers beeinflussbar $[28,40]$. Sie können deshalb in Abhängigkeit von der Diagnose vom Zahnarzt verordnet werden. Dabei sollte die Therapie von einem in der Behandlung von CMD erfahrenen und speziell weitergebildeten Physiotherapeuten vorgenommen werden (Abb. 18). Einheitliche Weiterbildungsangebote bzw. Weiterbildungsgänge in diesem Bereich sind bisher allerdings nicht definiert.

\subsection{Medikamentöse Therapie}

Die Möglichkeiten der medikamentösen Therapie wurden in der Vergangenheit oft nur wenig genutzt. Sie können in den meisten Fällen nur Teil eines Therapie-Gesamtkonzeptes sein. Indikationsgebiete sind Entzündungen, Arthralgien, Myalgien, chronische Schmerzen und damit sehr häufig verbundene Schlafstörungen. Nach Wirkprinzip unterschieden, können im Wesentlichen Analgetika, nonsteroidale Antirheumatika, Muskelrelaxantia, trizyklische Antidepressiva, Kortikoide sowie schlaffördernde Medikamente und Benzodiazepine zum Einsatz kommen. Hierbei ist zu beachten, dass der Patient schon aus forensischen Gründen über unerwünschte Wirkungen informiert werden muss, aber auch, um das Medikament bei leichteren, unerwünschten Wirkungen wie Müdigkeit nicht sofort abzusetzen. Des Weiteren sollte das Medikament gezielt nach Erkrankungssymptomen ausgewählt werden [41] und nicht nach dem Prinzip „one fits all“. Bei vielen Medikamenten, insbesondere Analgetika, ist darüber hinaus oftmals eine zeitregulierte Einnahme wirkungsvoller als die Einnahme nach Bedarf.

\subsection{Schienentherapie}

Okklusionsschienen sind aus zahnärztlicher Sicht das am häufigsten eingesetzte Therapiemittel, womit Symptome einer CMD je nach Zusammensetzung der untersuchten Patienten in ca. 60 bis $80 \%$ positiv beeinflusst werden [25,42-47]. Aus klinischer Sicht hat sich dabei eine Einteilung in Reflexschienen, Äquilibrierungsschienen und Positionierungsschienen bewährt. Sie orientiert sich an den bestehenden Symptomen, die betroffene Gewebsstrukturen (Zähne, Muskulatur, Kiefergelenk) berück-

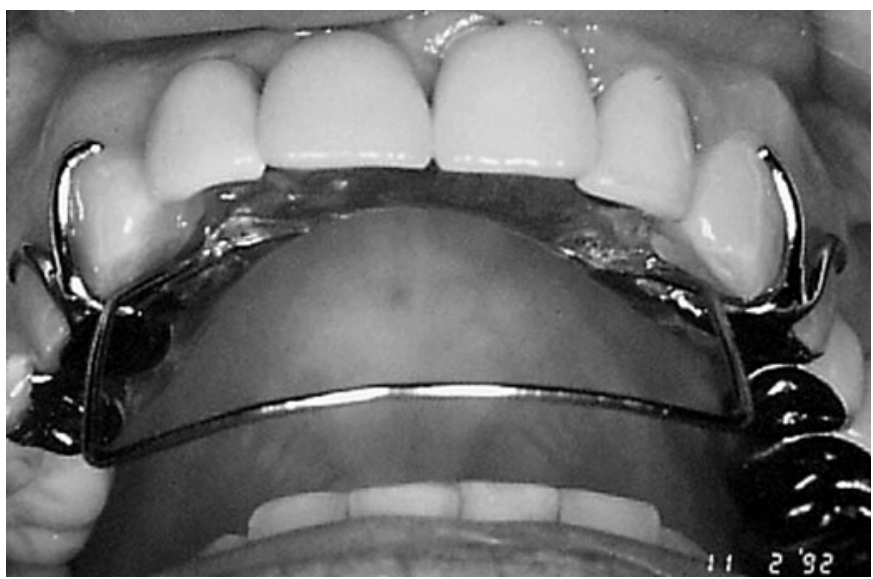

Abb. 19 Interzeptor nach Schulte.

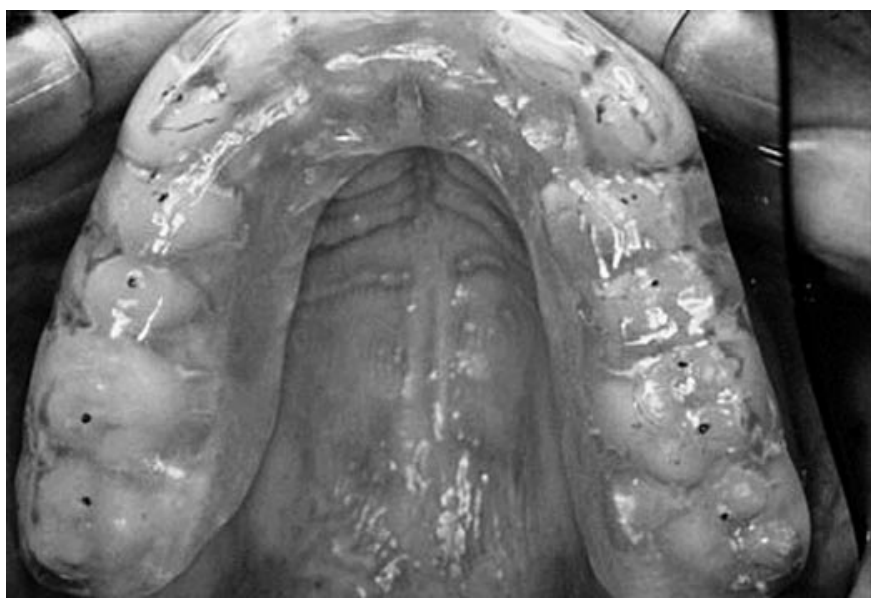

Abb. 20 Äquilibrierte Oberkiefer-Stabilisierungsschiene.

sichtigt und den Schweregrad der Erkrankung (akut/chronisch) mit einbezieht.

Reflexschienen (Interzeptor, Abb.19, [32], Miniplastschienen, anteriores Plateau) sind Okklusionsschienen, die den habituellen Zahnkontakt aufheben und damit Knirschen und Pressen verhindern und dadurch bestehende Zahn- und Muskelbeschwerden positiv beeinflussen. Reflexschienen sind indiziert bei akuten Beschwerden, die auf Überlastungen der beteiligten Gewebe zurückzuführen sind. Reflexschienen sind Kurzzeitschienen, da sie über die Aufhebung des Zahnkontaktes (therapeutischer Vorkontakt) selbst wieder Parafunktionen auslösen können. Deshalb ist ihre Tragezeit auf $8-14$ Tage zu begrenzen.

Äquilibrierungsschienen (Michigan-Schiene [48], Stabilisierungsschiene, Abb. 20, Zentrikschiene) sind Schienen, die eine ideale okklusale Beziehung herstellen, d. h. allseitiger, gleichmäBiger und gleichzeitiger Zahnkontakt in physiologischer Kondylenposition (Zentrik) in statischer Okklusion und eine Frontzahnführung mit Disklusion im Seitenzahngebiet in dynamischer Okklusion. Durch diese ideale Beziehung sollen Okklusionsstörungen, die zur Parafunktion geführt haben, ausgeschaltet werden, die Belastung für die beteiligten Gewebe minimiert werden und Stellungs- und Belastungsänderungen in den Kiefer- 


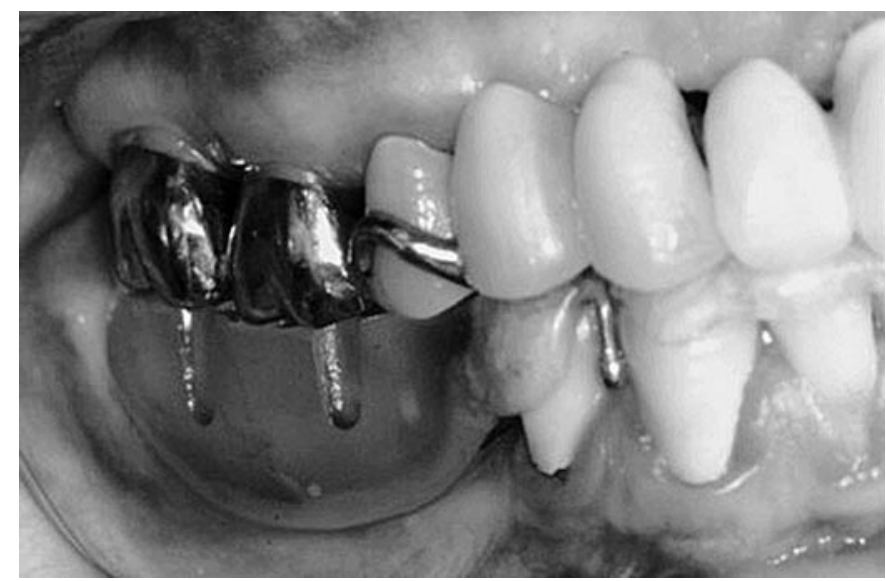

Abb. 21 Positionierungsschiene.

gelenken ausgeglichen werden. Äquilibrierungsschienen sind Kurzzeit- oder auch Langzeitschienen, sie können bei akuten und chronischen Beschwerden auch bei psychischen und physischen Überlastungsreaktionen eingesetzt werden. Je nach Ausgangssituation ist die Änderung der Kieferrelation durch diesen Schienentyp, auch nach längerer Tragezeit, gering. Die Äquilibrierungsschiene ist die am häufigsten zur Anwendung kommende Okklusionsschiene in der funktionellen Therapie.

Positionierungschienen (Repositionierungsschiene, Abb. 21, Dekompressionsschiene) sind Schienen, die zur Therapie von Kiefergelenkserkrankungen, wie einer anterior medialen Diskusverlagerung mit und ohne Reposition, Kiefergelenkkompression, Retralverlagerungen und Osteoarthrose eingesetzt werden $[25,49]$. Das Kiefergelenk oder beide Kiefergelenke wird bzw. werden durch die Schiene in einer therapeutischen Position eingestellt, um eine Heilung zu unterstützen und eine symptomund beschwerdefreie Gelenkbeziehung zu erhalten. Positionierungsschienen können als Kurz- oder Langzeittherapeutikum eingesetzt werden. Im Falle einer Kurzzeittherapie (bis zu 8 Wochen) wird die Schiene getragen bis eine deutliche Symptomlinderung eintritt. Sie wird danach in eine Äquilibrierungsschiene umgewandelt. Im Falle einer Langzeittherapie wird die Schiene getragen bis sich stabile Kiefergelenksfunktionen eingestellt haben. Sie sollte täglich 24 Stunden getragen werden was über mehrere Monate erforderlich sein kann. Speziell nach einer LangzeitPositionierungstherapie ist immer eine Rekonstruktion der okklusalen Kontaktbeziehung, sei es durch kieferorthopädische, prothetische oder kieferchirurgische Maßnahmen notwendig. Aus diesem Grunde muss die Indikation und Durchführung einer solchen Therapie besonders sorgfältig geplant und mit dem Patienten detailliert besprochen werden. Die Positionierungsschiene ist in der Kiefergelenktherapie allerdings oft das einzige Mittel, intrakapsuläre Verlagerungen auszugleichen und zu stabilisieren und somit die zugrunde liegenden pathologischen Veränderungen zu behandeln. Die Langzeitergebnisse hinsichtlich einer Behandlung von Schmerzen sind gut. Hingegen wird die Prognose für eine dauerhafte Beseitigung eines Gelenkknackens aufgrund einer anterioren Diskusverlagerung kontrovers beurteilt.

\subsection{Okklusale Therapie}

Als definitive funktionstherapeutische Maßnahmen werden das Einschleifen der Okklusion und die restaurative Versorgung zur Wiederherstellung harmonischer Zahnkontaktbeziehung angesehen [42,50]. Diese Maßnahmen sollten aber erst dann durchgeführt werden, wenn nach entsprechender Vorbehandlung stabile Verhältnisse, z.B. mit einer Äquilibrierungsschiene, hergestellt wurden. Die okklusale Korrektur (Einschleifen) natürlicher Zähne sollte nur dann vorgenommen werden, wenn Störungen sowohl klinisch als auch in der instrumentellen Okklusionsdiagnostik eindeutig verifiziert wurden. Das Einschleifen sollte schrittweise mit Sorgfalt durchgeführt werden und sich bei natürlichen Zähnen auf den Schmelz beschränken. Auch ist zu beachten, dass die Vertikaldimension durch die okklusale Adjustierung nicht zu stark abgesenkt wird. Ziel ist es, eine stabile Kontaktbeziehung in statischer Okklusion herzustellen. In dynamischer Okklusion sollte eine Front-Eckzahn-geschützte Okklusion oder Gruppenführung angestrebt werden. Restaurative Maßnahmen werden immer dann nach funktioneller Vorbehandlung notwendig, wenn Zähne fehlen, die habituelle Okklusion und/oder die Vertikaldimension verloren gegangen sind und wenn bestehende Störungen in statischer Okklusion nicht subtraktiv, also durch Einschleifmaßnahmen auszugleichen sind (Abb. 22-25) [25]. Oft ist die Indikation erst nach Auswertung

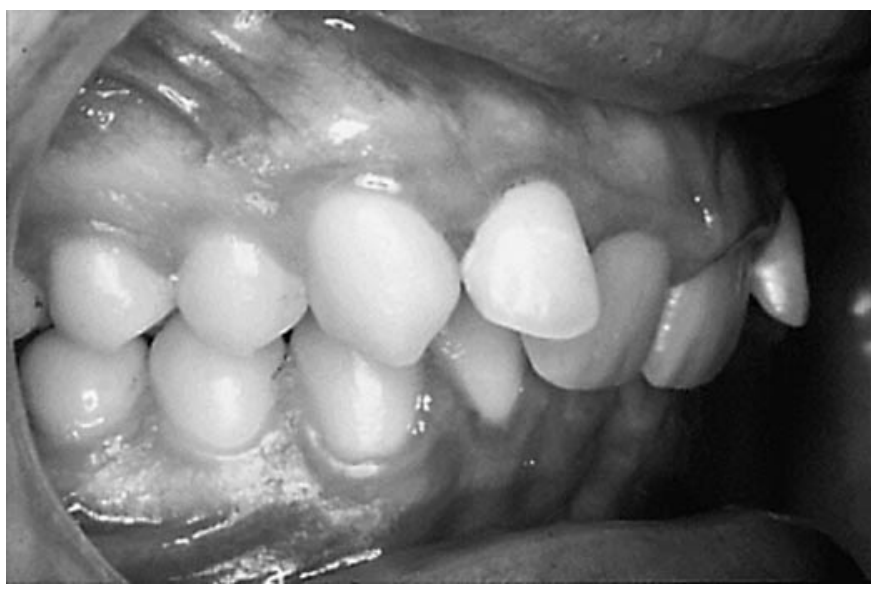

Abb. 22 Patientin mit Symptomen einer craniomandibulären Dysfunktion und einer Malokklusion.

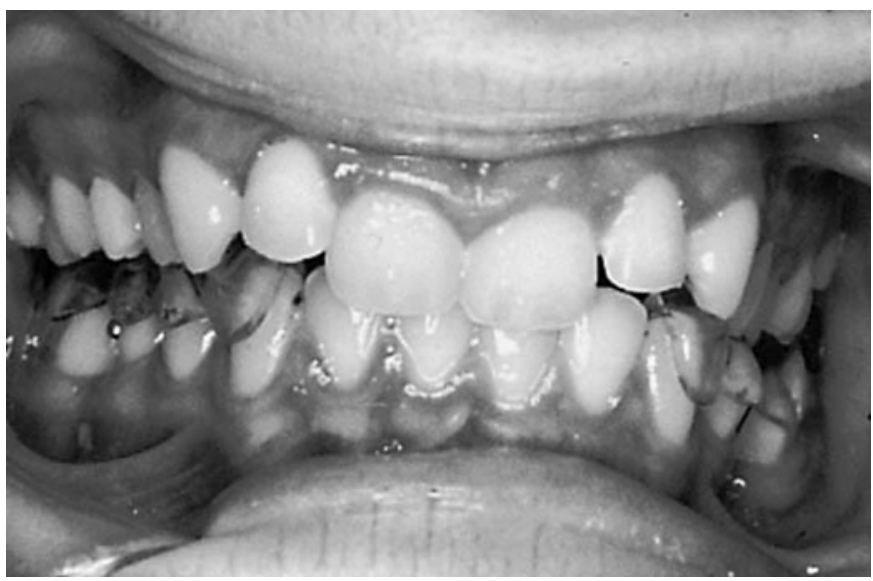

Abb. 23 Äquilibrierte Oberkiefer-Stabilisierungsschiene. 


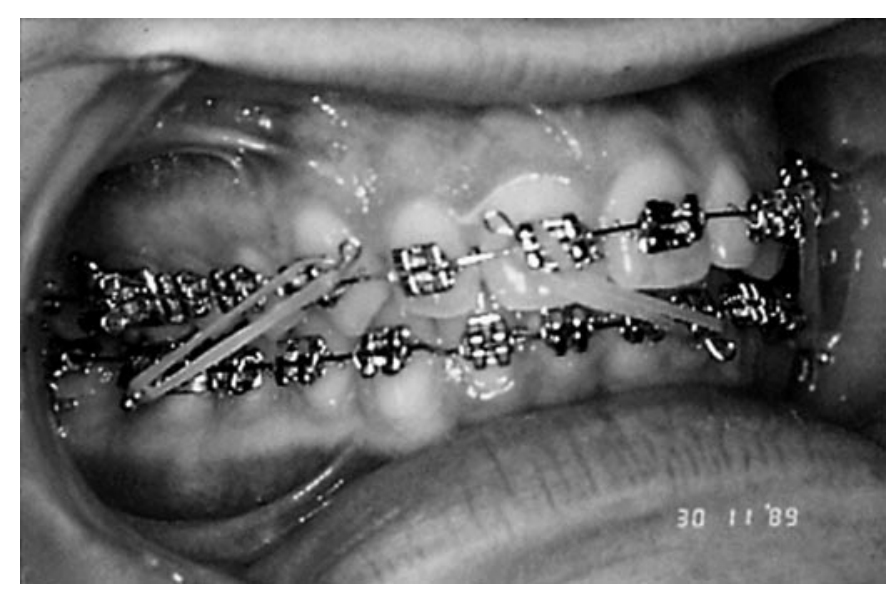

Abb. 24 Patientin während der kieferorthopädischen Behandlung.

der individuellen Situation über eine instrumentelle Okklusionsanalyse zu stellen. Ziel ist, wie beim Einschleifen, eine stabile Lagebeziehung zwischen Ober- und Unterkiefer in zentrischer Kondylenposition herzustellen. Es hat sich dabei klinisch gezeigt, dass es bei umfangreichen Versorgungen sinnvoll ist, die Rekonstruktionen 4 bis 6 Wochen Probe tragen zu lassen, um bei möglichen Lageverschiebungen des Unterkiefers zum Oberkiefer eine Neubeurteilung mittels einer erneuten Übertragung der neuen, zuvor probeweise getragenen Restaurationen in einen Kausimulator („Remontage“) durchführen zu können. Zu berücksichtigen ist, dass die Durchführung derartig restaurativ-funktionstherapeutischer Maßnahmen weit über den Schwierigkeitsgrad und Aufwand für „normale“ restaurative Behandlungen hinausgeht. Grundsätzlich sollte die Restauration regelmäßig kontrolliert werden und zudem nach Eingliederung der Restaurationen zu deren Schutz besonders bei weitergehenden Parafunktionen eine Äquilibrierungsschiene als Langzeitschiene eingegliedert werden.

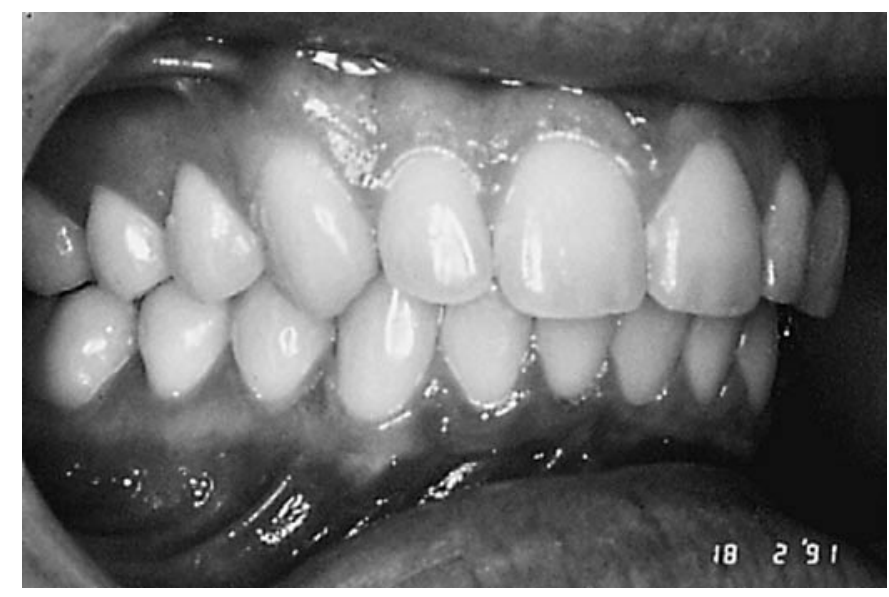

Abb. 25 Prothetische Neuversorgung und Wiederherstellung der Okklusion.

\section{$5 \quad$ Schlussfolgerung}

Abschließend kann gesagt werden, dass craniomandibuläre Dysfunktionen eine Gruppe von Erkrankungen darstellen, die oft vorübergehend und selbst limitierend sind und dabei meist keine gravierenden Auswirkungen im Hinblick auf Funktionseinschränkungen hinterlassen. Dies zeigt sich auch in epidemiologischen Untersuchungen, die eine Häufung der Erkrankung bei Patienten zwischen dem 20. und 50. Lebensjahr ermittelten. Danach scheint die Häufigkeit stark zurückzugehen. Vielleicht treten die Probleme durch schwerwiegendere Erkrankungen in den Hintergrund oder die beeinflussenden, uns heute noch vielfach unbekannten Faktoren nehmen ab. In jedem Fall sollte dieser Umstand dazu leiten, konservative und reversible Therapieformen, die der großen Mehrheit der Patienten innerhalb kurzer Zeit und auch für lange Zeit helfen, invasiveren und irreversiblen Therapien (Chirurgie, umfangreiche Rekonstruktionen etc.) vorzuziehen. Je früher eine adäquate Therapie beginnt, desto größer sind die Chancen auf einen raschen und dauerhaften Behandlungserfolg und desto geringer ist die Wahrscheinlichkeit der Entstehung therapieresistenter, chronischer Schmerzen. 


\section{Literatur}

${ }^{1}$ Türp JC, John M, Nilges P, Jürgens J, Ahlers MO, Böhner M, Busche E, Hugger A, Jakstat HA, Koch WH, Niederfeilner J, Paak S, Palatka P, Peschen-Rosin R, Schindler HJ, Sommer C, Sprotte G, Weißmann K, Wernze H. Schmerzen im Bereich der Kaumuskulatur und Kiefergelenke. Der Schmerz 2000; 14: 416-427

2 Palla S. Grundsätze zur Therapie des myoarthropathischen Schmerzes. Der Schmerz 2002; 16: 373 - 380

${ }^{3}$ Travell JG, Simons DG. Myofascial pain and dysfunction. The Trigger Point Manual. The upper Extremities. Baltimore: Williams \& Wilkins, 1983

${ }^{4}$ Bernhardt $O$ et al. Signs of temporomandibular disorders in tinnitus patients and in a population-based group of volunteers: results of the Study of Health in Pomerania. J Oral Rehab 2004; 31: 311 - 319

${ }^{5}$ Williamson EH. Interrelationship of internal derangement of the temporomandibular joint, headache, vertigo and tinnitus: a survey of 25 patients. Cranio 1990; 8 (4): 301 - 306

${ }^{6}$ Rubinstein B, Carlson GF. Effects of stomatognathic treatment of tinnitus: a retro-spective study. J Craniomandibular Pract 1987; 5: 254-259

${ }^{7}$ Bush FM. Tinnitus and otalgia in temporomandibular disorders. J Prosthet Dent 1987; 58 (4): 495-498

${ }^{8}$ Marasa FK, Ham BD. Case reports involving the treatment of children with chronic otitis media with effusion via craniomandibular methods. J Craniomandibular Pract 1988; 6: 256 - 270

${ }^{9}$ Loughner BA, Larkin LH, Mahan PE. Discomalleolar and anterior malleolar ligaments: possible causes of middle ear damage during temporomandibular joint surgery. Oral Surg, Oral Med, Oral Pathol 1989; 68 (1): $14-22$

${ }^{10}$ Ahlers MO, Freesmeyer WB, Göz G, Jakstat HA, Koeck B, Meyer G, Ottl $P$, Reiber T, Seeher WD. Klinische Funktionsanalyse (Stellungnahme der DGZMK und der AFDT). Zahnärztl Mitt 2003; 93: 1742

${ }^{11}$ Ahlers MO, Freesmeyer WB, Göz G, Jakstat HA, Koeck B, Meyer G, Ottl $\mathrm{P}$, Reiber T, Seeher WD. Instrumentelle, bildgebende und konsiliarische Verfahren zur CMD-Diagnostik (Stellungnahme der DGZMK und der AFDT). Zahnärztl Mitt 2003; 93: 1744

12 Jäger K, Graber G, Humar U. Die Therapie der dysfunktionellen Erkrankung des Kausystems. Dtsch Zahnärztl Z 1990; 45: 9

${ }^{13}$ Fuhr K, Reiber T. Klinische Funktionsdiagnostik. In: Koeck B (Hrsg). Funktionsstörungen des Kauorgans. Ausg. 8. München: Urban \& Schwarzenberg, 1995: 75

${ }^{14}$ Sassen H, Zeisler J, Windecker D. Zur Notwendigkeit klinischer Funktionsdiagnostik. Dtsch Zahnärztl Z 1985; 40: 177

15 Schwartz L, Chayes CM. The History and Clinical Examination. In: Schwartz L, Chayes CM (Hrsg). Facial Pain and Mandibular Dysfunction. Philadelphia: Saunders, 1968: 159

${ }^{16}$ Okeson JP. Management of temporomandibular disorders and occlusion. 4th ed. St. Louis, Missouri: Mosby-Year Book, Inc, 1998: 234 - 303

${ }^{17}$ Reiber T, Ottl P. Erfassungsbogen bei Craniomandibulären Dysfunktionen. Online-Formular zur Jahrestagung der Arbeitsgemeinschaft für Funktionslehre 2001. DGZMK URL: www.dgzmk.de/formulare/funktionsstatus.pdf, 2001

18 Ahlers MO, Jakstat HA (Hrsg). Klinische Funktionsanalyse. Interdisziplinäres Vorgehen mit optimierten Befundbögen. Mit Beiträgen von Ahlers MO, Danner HW, Gundlach KKH, Jakstat HA, Hugger A, Kopp S, Kordass B, Lamparter U, Peroz I, Sadjiroen A, Sander M, Türp JC und einem Geleitwort von Engelhardt JP, Hamburg: dentaConcept, 2000

${ }^{19}$ Engelhardt JP. Der klinische Funktionsstatus. Zahnärztl Mitt 1985; 75 : 420

${ }^{20}$ American Academy of Periodontology/American Dental Association. The Periodontal Screening Index. 1992

${ }^{21}$ Krogh-Poulsen WG, Olsson A. Management of the occlusion of the teeth, Part I: Background, definitions, rationale. In: Schwartz L, Chayes CM (Hrsg). Facial pain and mandibular dysfunction. Philadelphia: Saunders, 1968: 236

22 Gutowski A, Bauer A. Funktionsanalyse und Funktionstherapie im stomatognathen System. Berlin: Quintessenz, 1982

${ }^{23}$ Seligman DA, Pullinger AG. Association of occlusal variables among refined TM patient diagnostic groups. J Craniomandibular Disord 1989; 3 (4): $227-236$
${ }^{24}$ Pullinger AG, Seligman DA. Overbite and Overjet characteristics of refined diagnostic groups of temporomandibular disorder patients. Am J Orthod Dentofacial Orthop 1991; 100 (5): 401 - 415

${ }^{25}$ Freesmeyer WB. Zahnärztliche Funktionstherapie. München: Hanser, 1993

${ }^{26}$ Derogatis L. The SCL-90R manual-II: administration, scoring and procedures. Baltimore: Clinical Psychometric Research, 1983

${ }^{27}$ Franke H. Brief Symptom Inventory (BSI) von L. R. Derogatis - Deutsches Manual. Göttingen: Beltz Test Gesellschaft, 2000

${ }^{28}$ Bumann A. Funktionsdiagnostik und Therapieprinzipien. Stuttgart: Georg Thieme Verlag, 2000

${ }^{29}$ Vogl TJ. MR - Tomographie am Temporomandibulargelenk. Stuttgart: Thieme Verlag, 1993

${ }^{30}$ Laskin DM. Etiology of the pain-dysfunction-syndrome. J Am Dent Assoc, 1969; 79 (1): 147-153

31 Schulte W. Zur funktionellen Behandlung der Myo-Arthropathien des Kauorgans: Ein diagnostisches und physiotherapeutisches Programm. Dtsch Zahnärztl Z 1970; 25 (3): 422 - 436

${ }^{32}$ Schulte W. Die exzentrische Okklusion. Berlin: Quintessenz, 1983

${ }^{3}$ Ahlers MO, Jakstat HA, Freesmeyer WB, Simonis A, Hugger A, Meyer G. Vorschlag eines modernen Diagnoseschemas zur therapiespezifischen Erfassung von Anamnesen und Befunden bei CMD (Online-Abstract zur Jahrestagung der Arbeitsgemeinschaft für Funktionslehre 2001). DGZMK, URL: http://www.dgzmk.de/set2.htm

${ }^{34}$ Center for Disease Control and Prevention/National Center for Health Statistics. Classification of Diseases. U.S. Department of health and human services; Division of Data Services, 2000

${ }^{35}$ American Academy of Orofacial Pain Guidelines Committee, edited by Okeson, JP. Diagnostic Classification of Orofacial Pain Disorders. In: The American Academy of Orofacial Pain (Hrsg). Orofacial Pain Guidelines for Assessment, Classification, and Management. Coral Stream, Illinois: Quintessence, 1996: 45

${ }^{36}$ Headache Classification Committee of the International Headache Society, edited by Olesen J. Classification and diagnostic criteria for headache disorders, cranial neuralgias and facial pain. Cephalalgia, 1988; 8 (Suppl 7)

37 Palla S, Koller M. Die Therapie der Myoarthropathien des Kausystems. Zürich: Eigenverlag, 1989

38 Jäger K. Streßbedingte Kaufunktionsstörungen. Berlin: Quintessenz Verlag, 1997

${ }^{39}$ Freesmeyer WB. Streßbedingte Kaufunktionsstörungen. Dtsch Zahnärztl Z 1997; 52: 514ff

${ }^{40}$ Hansson TL, Honee W, Hesse J. Funktionsstörungen im Kauorgan. Heidelberg: Hüthig, 1990

${ }^{41}$ Fussnegger MF. Medikamentöse Therapie bei Craniomandibulären Dysfunktionen. Zahnärztl Mitt 2004; 94 (22): 62 - 70

42 Koeck B. Rekonstruktive Maßnahmen. In: Hupfauf L (Hrsg). Funktionsstörungen des Kauorgans. Praxis der Zahnheilkunde 8. München: Urban \& Schwarzenberg, 1995

${ }^{43}$ Lotzmann U. Okklusionsschienen und andere Aufbißbehelfe. 3. Aufl. München: Neuer Merkur, 1992

${ }^{44}$ Ottl P, Lauer HC. Aufbißschienentherapie bei Funktionsstörungen. Zahnärztl Prax 1991; 42 (12): 458-460

${ }^{45}$ Bumann A, Kopp S, Stangohr M, Rahlf B. Der Stellenwert der Aufbißschienentherapie bei Funktionsstörungen im stomatognathen System. Dtsch Zahnärztl Z 1989; 44: 14

${ }^{46}$ Ferrario VF, Sforza C, Tartaglia GM, Dellavia C. Immediate effect of a stabilization splint on masticatory muscle activity in temporomandibular disorder patients. J Oral Rehabil 2002; 29 (9): 810-815

${ }^{47}$ Gavish A, Winocur E, Ventura YS, Halachmi M, Gazit E. Effect of stabilization splint therapy on pain during chewing in patients suffering from myofascial pain. J Oral Rehabil 2002; 29: 1181

${ }^{48}$ Ash MM, Schmidseder J. Schienentherapie. München, Jena: Urban \& Fischer Verlag, 1999

${ }^{49}$ Stegenga B, Dijkstra PU, de Bont LG, Boering G. Temporomandibular joint osteoarthrosis and internal derangement. Part II: Additional treatment options. Int Dent J 1990; 40 (6): 347 - 353

${ }^{50}$ Hanel G. Das Einschleifen. In: Hanel G (Hrsg). Funktionsanalytisch orientierte Praxis. Berlin: Quintessenz, 1984: 207 